\title{
Linear shear and nonlinear extensional rheology of unentangled supramolecular side- chain polymers
}

Cui, Guanghui; Boudara, Victor A. H.; Huang, Qian; Baeza, Guilhem P.; Wilson, Andrew J.; Hassager, Ole; Read, Daniel J.; Mattsson, Johan

Published in:

Journal of Rheology

Link to article, DOI:

$10.1122 / 1.5012349$

Publication date:

2018

Document Version

Publisher's PDF, also known as Version of record

Link back to DTU Orbit

Citation (APA):

Cui, G., Boudara, V. A. H., Huang, Q., Baeza, G. P., Wilson, A. J., Hassager, O., Read, D. J., \& Mattsson, J. (2018). Linear shear and nonlinear extensional rheology of unentangled supramolecular side-chain polymers. Journal of Rheology, 62(5), 1155-1174. https://doi.org/10.1122/1.5012349

\section{General rights}

Copyright and moral rights for the publications made accessible in the public portal are retained by the authors and/or other copyright owners and it is a condition of accessing publications that users recognise and abide by the legal requirements associated with these rights.

- Users may download and print one copy of any publication from the public portal for the purpose of private study or research.

- You may not further distribute the material or use it for any profit-making activity or commercial gain

- You may freely distribute the URL identifying the publication in the public portal 


\section{Linear shear and nonlinear extensional rheology of unentangled supramolecular side- chain polymers}

Guanghui Cui, Victor A. H. Boudara, Qian Huang, Guilhem P. Baeza, Andrew J. Wilson, Ole Hassager, Daniel J. Read, and Johan Mattsson

Citation: Journal of Rheology 62, 1155 (2018); doi: 10.1122/1.5012349

View online: https://doi.org/10.1122/1.5012349

View Table of Contents: http://sor.scitation.org/toc/jor/62/5

Published by the The Society of Rheology

\section{Articles you may be interested in}

Rheo-NMR of transient and steady state shear banding under shear startup

Journal of Rheology 62, 1125 (2018); 10.1122/1.5037594

The transient behavior of soft glassy materials far from equilibrium

Journal of Rheology 62, 869 (2018); 10.1122/1.5024701

CaBER vs ROJER-Different time scales for the thinning of a weakly elastic jet

Journal of Rheology 62, 1135 (2018); 10.1122/1.5021834

Computing the linear viscoelastic properties of soft gels using an optimally windowed chirp protocol Journal of Rheology 62, 1037 (2018); 10.1122/1.5018715

Rheological behaviors of $\mathrm{H}$-shaped polymers incorporated with short branches under shear and elongational flows via FENE-Rouse model

Journal of Rheology 62, 1115 (2018); 10.1122/1.5026530

Shear thinning in dilute and semidilute solutions of polystyrene and DNA

Journal of Rheology 62, 845 (2018); 10.1122/1.5010203

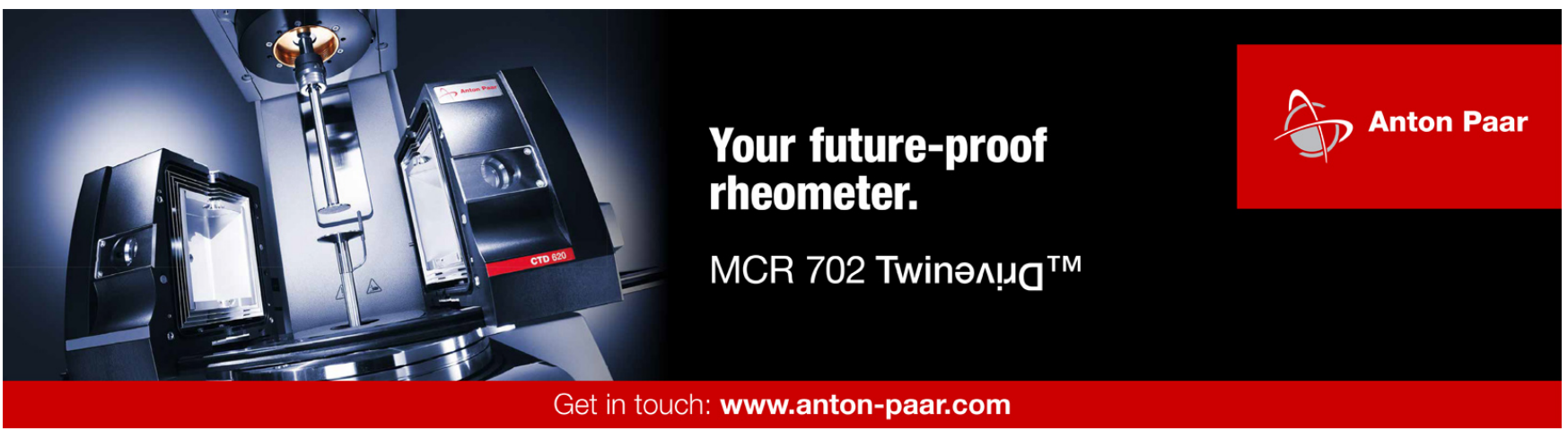




\title{
Linear shear and nonlinear extensional rheology of unentangled supramolecular side-chain polymers
}

\author{
Guanghui Cui, ${ }^{1}$ Victor A. H. Boudara, ${ }^{2}$ Qian Huang, ${ }^{3}$ Guilhem P. Baeza, ${ }_{1}^{4}$ Andrew J. Wilson,,${ }^{5,6}$ Ole Hassager, ${ }^{3}$ \\ Daniel J. Read, ${ }^{2}$ and Johan Mattsson ${ }^{1, a)}$ \\ ${ }^{1}$ School of Physics and Astronomy, University of Leeds, Leeds LS2 9JT, United Kingdom \\ ${ }^{2}$ School of Mathematics, University of Leeds, Leeds LS2 9JT, United Kingdom \\ ${ }^{3}$ Department of Chemical and Biochemical Engineering, Technical University of Denmark, Lyngby, Denmark \\ ${ }^{4}$ University of Lyon, INSA-Lyon, CNRS, MATEIS, UMR 5510, F-69621, Villeurbanne, France \\ ${ }^{5}$ School of Chemistry,University of Leeds, Woodhouse Lane, Leeds LS2 9JT, United Kingdom \\ ${ }^{6}$ Astbury Center for Structural Molecular Biology, University of Leeds, Woodhouse Lane, Leeds LS2 9JT, United \\ Kingdom
}

(Received 6 November 2017; final revision received 26 June 2018; published 10 August 2018)

\begin{abstract}
Supramolecular polymers are important within a wide range of applications including printing, adhesives, coatings, cosmetics, surgery, and nano-fabrication. The possibility to tune polymer properties through the control of supramolecular associations makes these materials both versatile and powerful. Here, we present a systematic investigation of the linear shear rheology for a series of unentangled ethylhexyl acrylate-based polymers for which the concentration of randomly distributed supramolecular side groups is systematically varied. We perform a detailed investigation of the applicability of time temperature superposition (TTS) for our polymers; small amplitude oscillatory shear rheology is combined with stress relaxation experiments to identify the dynamic range over which TTS is a reasonable approximation. Moreover, we find that the "sticky-Rouse" model normally used to interpret the rheological response of supramolecular polymers fits our experimental data well in the terminal regime, but is less successful in the rubbery plateau regime. We propose some modifications to the "sticky-Rouse" model, which includes more realistic assumptions with regard to (i) the random placement of the stickers along the backbone, (ii) the contributions from dangling chain ends, and (iii) the chain motion upon dissociation of a sticker and reassociation with a new co-ordination which involves a finite sized "hop" of the chain. Our model provides an improved description of the plateau region. Finally, we measure the extensional rheological response of one of our supramolecular polymers. For the probed extensional flow rates, which are small compared to the characteristic rates of sticker dynamics, we expect a Rouse-type description to work well. We test this by modeling the observed strain hardening using the upper convected Maxwell model and demonstrate that this simple model can describe the data well, confirming the prediction and supporting our determination of sticker dynamics based on linear shear rheology. (C) 2018 The Society of Rheology.

https://doi.org/10.1122/1.5012349
\end{abstract}

\section{INTRODUCTION}

Supramolecular polymers are made of covalent chains connected through reversible interactions, such as hydrogen bonding [1-25], metal-ligand coordination [26-38], and ionic aggregation [39-45]. The ability to vary and control the interactions in supramolecular systems provides an efficient tool to tune the structure, dynamics, and rheology [26,29,46]. Among the possible supramolecular interactions, quadruple hydrogen bonding groups, 2-ureido-4[1H]-pyrimidinone (UPy), were chosen in this study since their properties and behavior with regard to chemical synthesis are well understood $[19,47]$. The UPy groups are characterized by a strong association constant $\left(k_{\text {assoc }}>10^{6} \mathrm{M}^{-1}\right.$ in chloroform) [16], leading to significant effects on material properties, and the hydrogen (H-) bonding nature of UPy interactions leads to

\footnotetext{
a) Author to whom correspondence should be addressed; electronic mail: k.j.l. mattsson@leeds.ac.uk
}

interesting and useful temperature sensitivity of the interactions $[23,48]$.

Supramolecular polymers based on UPy groups have been widely investigated and materials with important characteristics such as stimuli-responsive [23], self-healing [10,11,4952], and temperature responsive [53,54] properties have found applications within printing [55-58], cosmetics [59,60], adhesives [61], and coatings [62]. As an example of how supramolecular associations can play an important role, for inkjet printing applications a UPy-modified polyether mixed with stabilizers, antioxidants, and colorants was used in the work by Jaeger et al. [55]. The ink needs a low viscosity during droplet ejection, but should be highly viscous or even solid once it is deposited on the print surface. The supramolecular associations here ensure the solid-like nature of the printed ink at ambient temperatures, but the elevated temperatures during deposition dissociate the network leading to the low deposition viscosity. Generally, it is thus important to understand the rheological response for a proper control of the material behavior. The effect of UPy addition on the linear viscoelasticity of supramolecular polymers has 
previously been investigated [20,63-66]. However, few studies exist where the rheological response is characterized for a systematic variation of supramolecular side-group density $[41,66,67]$.

Time temperature superposition (TTS) is a commonly used and often powerful method to evaluate the linear rheological properties of a material over a wide time or frequency window [68]. TTS is based on the assumption that the underlying friction coefficient for all relevant relaxation processes (segmental as well as chain relaxation including Rouse and/ or reptation mechanisms) is the same, that is to say, these relaxation processes all arise as the summation of increments of the same "local" motions and, therefore, are accelerated or retarded by the same factor as temperature is varied [68]. A material for which TTS is applicable is termed "thermorheologically simple" [69]. Although TTS is commonly used, it is well known that TTS breaks down for many polymers because of the different temperature dependence of segmental and chain relaxation processes [70-74]. A supramolecular polymer is characterized by at least two types of interactions: the van der Waals attraction (friction effect) and additional supramolecular interactions. The chain motions associated with these two interactions, respectively, can be expected to behave differently as temperature is varied leading to a breakdown of TTS. To what degree TTS still approximately holds for a particular supramolecular system will depend on the specific material and interaction details and it is not uncommon for TTS to be applied to supramolecular systems [20,63-67,70,75], even though alternative techniques that permit data to be obtained over extended dynamic range can preclude the use of TTS [76].

Several theoretical models have been proposed to describe the rheological response of telechelic polymers $[77,78]$ and unentangled [41,79-82] or entangled [83-87] supramolecular polymers. For unentangled polymers with supramolecular side groups, the polymer type relevant to our work, the so-called sticky-Rouse model has been proposed [41,79-82]. Here, the standard Rouse model for single chain dynamics is modified to take into account the effects of the sticker interactions on the viscoelastic properties of the supramolecular material; the associations and dissociations of the sticky groups are assumed to act as an extra friction between polymer chains and thus to delay the terminal relaxation. The sticky-Rouse model can be generalized to account both for polydispersity in the overall molecular weight and for variation in the total number of stickers per chain [65]. The model has been used to describe data on supramolecular polymers and it typically fits the experimental data quite well in the terminal regime $[41,65,88]$. However, a relatively large mismatch between data and theory can often be observed in the rubber plateau region [41,65], and we find the same to be true for the polymers in the present investigation.

One reason for the mismatch is that, in the sticky-Rouse model, the supramolecular groups are assumed to be evenly distributed along the chain. More precisely, the slowest modes of the Rouse spectrum are assumed to be uniformly retarded by the sticky group timescale, without changing their essential mode distribution, while the faster modes are left as is; this is closely equivalent to assuming an even distribution of stickers along the chain. For our polymers we need to relax this hypothesis because (i) our four supramolecular polymers have a relatively low sticker concentration (2, 6, 9, and $14 \mathrm{~mol} . \%)$ and (ii) random copolymerization implies a random placement of the stickers along the backbone. Moreover, (iii) the contribution to the response from dangling chain ends, which differs from the relaxation modes of segments of chain "trapped" between stickers, is not considered in the common formulation of the sticky-Rouse model, and finally (iv) the chain motion upon dissociation of a sticker and reassociation with a new coordination involves a finite sized "hop" of the chain, rather than a continuous motion with increased friction, as assumed in the standard sticky-Rouse model. We find that our model can fit data precisely in the terminal region and improves the fit in the rubber plateau region. However, while we have included some extra and essential details in our model, we still find that the fit is not perfect, especially for samples with higher sticker concentration. We provide a discussion regarding what elements might still be missing from the model, to provide a full description of the rheology.

The relevant deformation and flow conditions during polymer processing is often of extensional character. However, for supramolecular polymers with side-chain functional groups, relatively few studies have been reported [89-91]. As an example, Shabbir and co-workers [91] have reported the extensional rheology of poly(butyl acrylate-coacrylic acid) with varying acrylic acid content. H-bonds can form between acrylic acid groups and introduce chain-chain interactions. Strain hardening was observed for strain rates significantly smaller than the inverse of the reptation time, indicating that the strain hardening for their studied polymer system is attributed to stretching of chain segments which are restricted by hydrogen bonding groups. Similar observations were also made for ionomers $[89,90]$. In ionomers, the supramolecular interactions originate from association of ionic groups covalently attached to either the polymer backbone or the side groups [92]. Associations between these ionic groups typically lead to nanometer-sized aggregates which act as physical cross-links. The magnitude of the strain hardening in ionomers was related to the strength of these ionic clusters and a stronger cohesive strength of the ionic clusters leads to a more pronounced strain hardening. The ionic aggregates of ionomers thus correspond to chain-chain interactions via H-bonds for our UPy-based supramolecular polymers.

In the present paper, we present a detailed investigation of the rheological response of a series of linear polymers in which the concentration of randomly distributed supramolecular side groups is systematically varied. A homo-polymer, poly(ethylhexyl acrylate) (PEHA) and four copolymers (UPyPEHAx) composed of ethylhexyl acrylate and 2-(3-(6-methyl-4-oxo-1,4-dihydropyrimidin-2-yl)ureido)-ethyl acrylate (UPyEA) with varying concentrations of UPyEA $\left(\phi_{\mathrm{UPy}}\right)$ of $2,6,9$, and 14 mol. $\%$, respectively, are synthesized using RAFT polymerization [93,94], see Fig. 1. The letter " $\mathrm{x}$ " in the abbreviation indicates different concentrations of $\phi_{\mathrm{UPy}}$ expressed in mol. \%. We are not aware of any studies that have determined the entanglement or critical molecular weight of PEHA. However, a comparable acrylate 

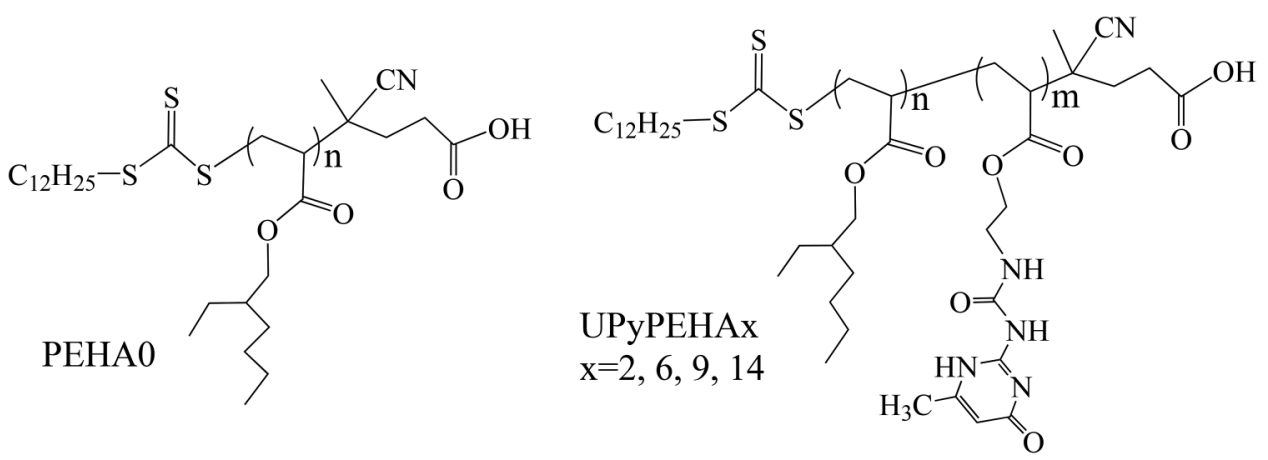

FIG. 1. Chemical structures of PEHA and UPyPEHA with varying $\phi_{\mathrm{UPy}}$. The letter " $\mathrm{x}$ " in the figure indicates the $\phi_{\mathrm{UPy}}=2,6,9,14 \mathrm{~mol}$. $\%$.

polymer with a linear side chain also containing eight carbons, poly octyl acrylate (POA), has an $M_{\mathrm{e}} \sim 15 \mathrm{~kg} / \mathrm{mol}$ and an estimated $M_{\mathrm{c}} \sim 25 \mathrm{~kg} / \mathrm{mol}$ [95]. The molecular weights of all our samples (Table I) are below this $M_{\mathrm{c}}$ and we thus expect our polymers to be unentangled, meaning that the cross-linking effects of reversible supramolecular sidegroup interactions can be readily identified. We focus on four particular aspects of the rheology of our samples: (i) the effects of adding UPy-based side groups on the linear viscoelasticity, (ii) a detailed investigation of the extent to which TTS can be applied to our series of polymers, where we complement our small amplitude oscillatory shear (SAOS) experiments with measurements of stress relaxation resulting from a step shear strain; here, the time-dependent response is converted to the frequency domain to extend the frequency range accessed at a single temperature, (iii) detailed modeling of the linear rheological response using both a standard and a modified version of the sticky-Rouse model, and (iv) extensional rheology measurements on one of our supramolecular polymers, UPyPEHA6, together with modeling using a simple upper convected Maxwell modeling which is expected to be applicable for extensional flow rates where Rouse-like dynamics are relevant.

\section{EXPERIMENTAL SECTION}

Five polymers were synthesized by RAFT polymerization, see Table I. The first homo-polymer (PEHA0) was

TABLE I. Characteristics of (co-)polymers with varying UPy contents, $\phi_{\mathrm{UPy}}$.

\begin{tabular}{|c|c|c|c|c|c|}
\hline Sample codes & $\begin{array}{c}M_{\mathrm{n}} \\
(\mathrm{kg} / \mathrm{mol})^{\mathrm{a}}\end{array}$ & $\mathrm{PDI}^{\mathrm{a}}$ & $\begin{array}{l}\text { UPy ratio } \\
(\text { mol. \% })^{\text {b }}\end{array}$ & $n(\mathrm{UPy})^{\mathrm{c}}$ & $n(\mathrm{EHA})^{\mathrm{c}}$ \\
\hline PEHA0 & 17.2 & 1.05 & - & - & - \\
\hline UPyPEHA2 & 16.6 & 1.24 & 2 & 2 & - \\
\hline UPyPEHA6 & 22.0 & 1.38 & 6 & 7 & 16 \\
\hline UPyPEHA9 & 23.7 & 1.71 & 9 & 11 & 10 \\
\hline UPyPEHA14 & 24.6 & 2.26 & 14 & 17 & 6 \\
\hline
\end{tabular}

${ }^{a}$ Measured by SEC calibrated with polystyrene standards in THF.

${ }^{\mathrm{b}}$ Measured by NMR.

${ }^{c}$ Average number of the UPyEA per chain calculated based on SEC and NMR results.

${ }^{\mathrm{d}}$ Average number of EHA monomers between two UPyEA groups calculated based on SEC and NMR results. synthesized from ethylhexyl acrylate (EHA). The other four copolymers were synthesized from EHA and 2-(3(6-methyl-4-oxo-1,4-dihydropyrimidin-2-yl)ureido)-ethyl acrylate (UPyEA) with systematically increasing concentrations of UPyEA. The chemical structures of the polymers are shown in Fig. 1. Some key characteristics of the samples, including their number average molecular weight $M_{\mathrm{n}}$, their polydispersity indices (PDI), the number of UPyEA side groups per chain, and the average number of EHA monomers between UPyEA side groups are listed in Table I. Two UPy groups are interacting through the formation of quadruple hydrogen bonds, as shown in the sketch in Fig. 1 and thus dimers of interacting UPy groups, lead to reversible supramolecular associations and hence a transient network of polymer chains.

SAOS measurements and step strain stress relaxation experiments were performed using a rheometrics advanced expansion system (ARES) strain-controlled rheometer equipped with two complementary force rebalance transducers. The experiments were conducted within a temperature range from $T_{\mathrm{g}}(\approx 203$ to $215 \mathrm{~K})$ to $T=403 \mathrm{~K}$ using a convection oven operating under nitrogen flow with a temperature control better than $\pm 0.5 \mathrm{~K}$. A plate-plate geometry was used in the experiments and either 3- or 10-mm diameter parallel plates were used depending on the composition of the samples and on the testing temperature. Polymer films with a thickness of about $1.5 \mathrm{~mm}$ were obtained by placing the polymers in a round mold at $T=403 \mathrm{~K}$ under vacuum for 3 days. The films were placed between the rheometer plates and their edges were trimmed to match the geometry. For each sample, strain sweep tests were carried out to ensure that the measurements were performed within the linear range. For the range of determined material moduli, we confirmed that our results are not influenced by a variation of plate diameters $(3,5$, and $10 \mathrm{~mm}$ plates $)$ and thus recorded torques, demonstrating that we are not influenced by instrument compliance effects [41].

For the oscillatory shear experiments, the complex shear modulus $\left(G^{*}=G^{\prime}+i G^{\prime \prime}\right)$ was determined over an angular frequency range of $0.628-62.8 \mathrm{rad} / \mathrm{s}$. To obtain the rheological response over a wider frequency range, TTS using horizontal shift factors was used. We note that vertical shifts are also often used to account for the temperature variation of the density. However, with the quantities of the polymers available in this work, we could not reliably determine the 
temperature-dependent densities and thus chose to use only horizontal shift factors. To investigate the accuracy of this approach we plot the loss tangent $\tan (\delta)$ vs the complex modulus $\left|G^{*}\right|$ in a so-called Van-Gurp-Palmen (VGP) plot [96,97] in Fig. 2. This representation removes all explicit time-dependence from the data, and so indicates whether an accurate frequency-shift TTS is possible or not. Based on this plot, we find that for the data where TTS works well, as determined from our detailed analysis described in the Results and Discussion section, master curves are formed without the need for vertical shifts, thus supporting our approach of only using horizontal shift factors.

TTS was initially conducted using the software, Orchestrator from TA instrument, which takes both $G^{\prime}$ and $G^{\prime \prime}$ into account in the optimization of the data shifting. For the samples with 0,2 , and 6 mol. \% UPy groups, TTS was solely performed using this procedure. For the samples with 9 and 14\% UPy groups, the initial optimization was performed in the same manner, subsequently followed by small manual horizontal adjustments $(\leq 10 \%)$ aimed to result in a continuous $G^{\prime}$ curve. Also, as further described below, we demonstrate that when TTS works well, the results are fully consistent with those resulting from stress relaxation measurements, which cover a wider frequency range without need for TTS. This further supports the fact that the introduction of a vertical shift factor is not necessary within the accuracy of the experiments for our polymers.

In the stress relaxation experiments, a step strain with a rise time of 0.01 to $0.1 \mathrm{~s}$ within the linear regime was applied to the material and this strain subsequently remained constant over time. The stress responding to the applied strain was recorded as a function of time. In practice, before the real test, a small pre-strain was applied to the material which aims to eliminate the effect of pre-stress in the material and improve the experiment reproducibility. The waiting time for the pre-strain to relax should be long enough so that the stress resulting from the applied strain is negligible. The software, iRheo, was used to perform the transformation from time-dependent stress relaxation data to frequency-dependent dynamic moduli [98]. The mathematical approach used by
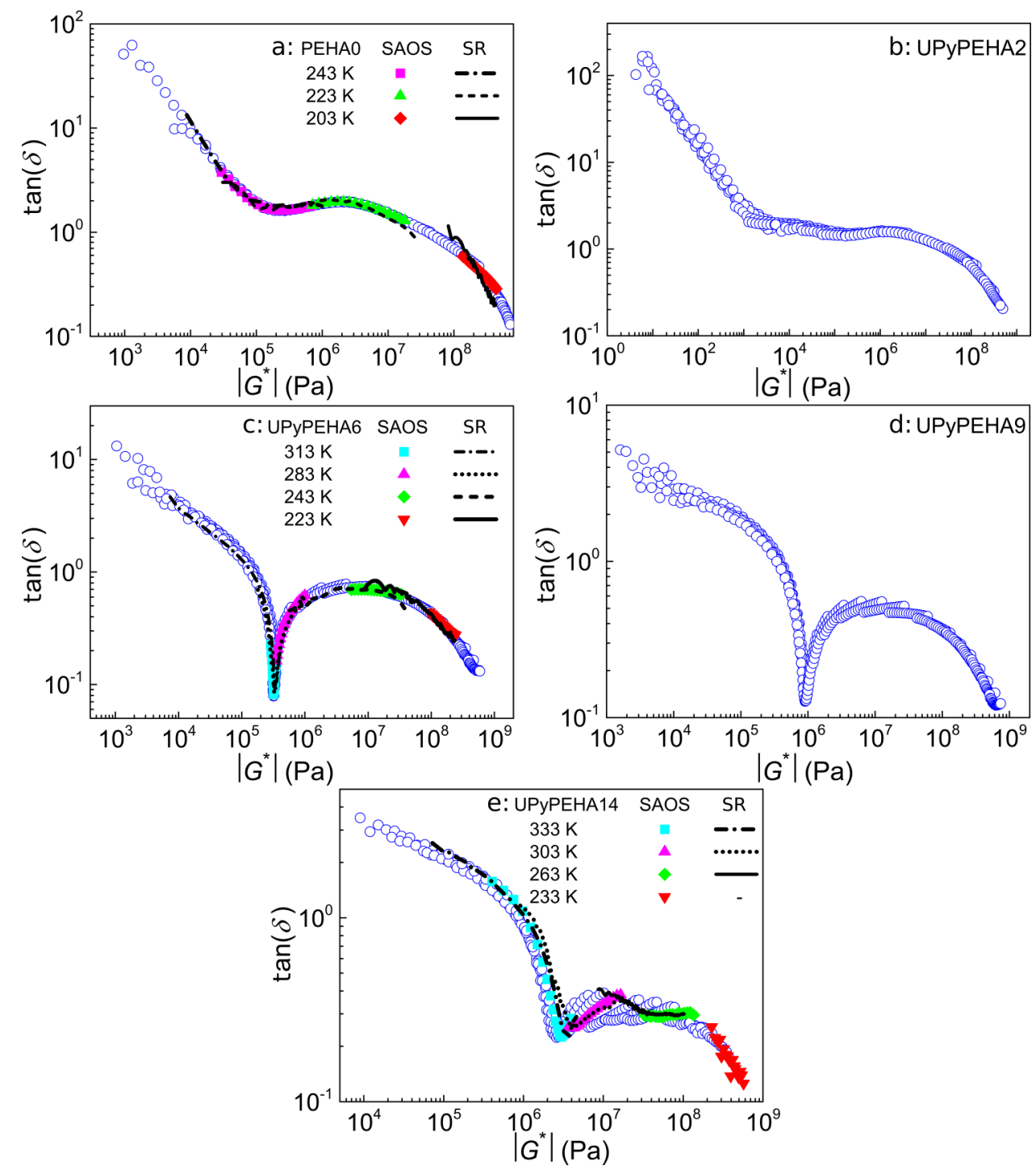

FIG. 2. Plots of $\tan (\delta)$ as a function of $\left|G^{*}\right|$ for all samples. The SAOS data are shown in blue circles. For the temperatures where stress relaxation measurements are also performed, the SAOS data are shown using colored symbols, as described in the legends of panels a, c, and e. The corresponding black lines show the results calculated from the stress relaxation (SR) data. 

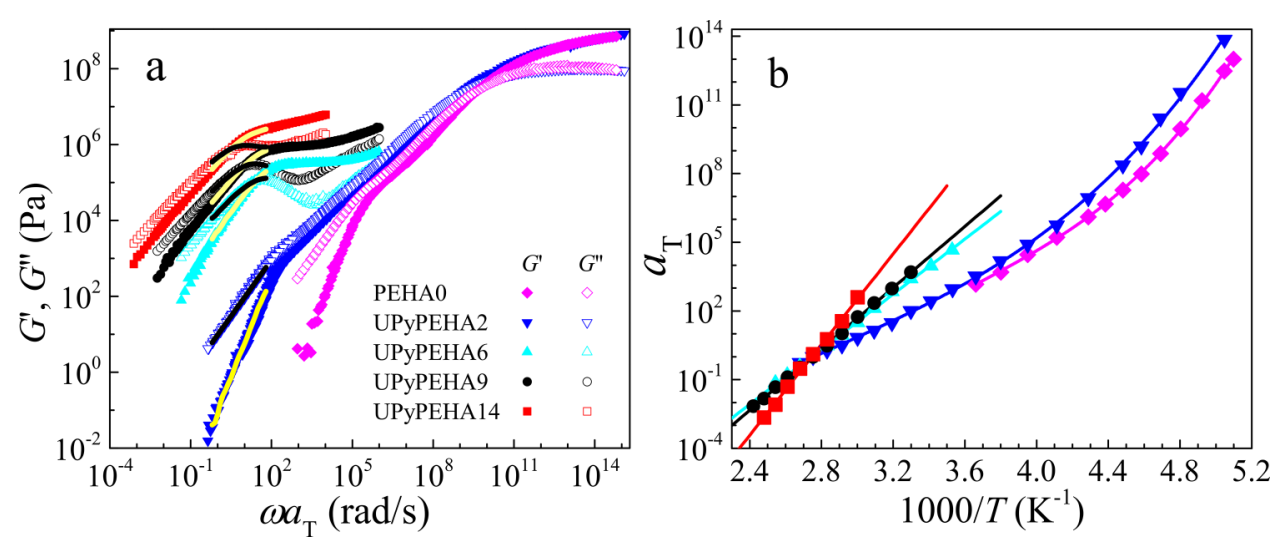

FIG. 3. (a) TTS master curves for the polymer samples with varying $\phi_{\mathrm{UPy}}$ at a reference temperature of $T=363 \mathrm{~K}$ for the four UPyPEHA samples. Since the torque at $T=363 \mathrm{~K}$ is too small for the SAOS experiment on the PEHA0 polymer sample, the master curve for the PEHA0 sample was created using an initial reference temperature of $T=263 \mathrm{~K}$, and the determined shift factors were subsequently extrapolated to $T=363 \mathrm{~K}$ so that all data shown in the figure could be displayed at effectively the same reference temperature of $T=363 \mathrm{~K}$ to facilitate comparisons. The master curves are constructed only using data for which we determined that TTS is a good approximation (see the discussion in the text). The black and yellow solid lines mark the SAOS data at the reference temperature. (b) The temperature-dependent shift factors $a_{T}$ used to construct the master curves in panel a. The shift factors for PEHA0 and UPyPEHA2 are fitted using a WLF expression, whereas the other polymer data are fitted using an Arrhenius expression.

iRheo performs the transformation without the use of fitting functions and has the significant advantage that it takes account of the response also from the initial strain ramp period and thus extends the frequency range of the transformed moduli. The interpretation of the output from iRheo and the assessment of its accuracy are discussed later.

In the extensional rheology experiments, the timedependent extensional stress growth coefficient (i.e., stress divided by strain rate, $\sigma / \dot{\varepsilon}$ ) was measured using a filament stretching rheometer (DTU-FSR) [99]. Cylindrical stainless steel sample plates with a diameter of $5.4 \mathrm{~mm}$ were used for the measurements. The latter were performed at a constant Hencky strain rate, $\dot{\varepsilon}$, imposed at the mid-filament diameter using a real-time control software. The time-dependent Hencky strain, $\varepsilon$, is defined as: $\varepsilon(t)=-2 \ln \left(R(t) / R_{0}\right)$, where $R(t)$ and $R_{0}$ are the radii of the filament at times $t$ and 0 , respectively. The rheometer can be operated over the temperature range with an accuracy of $\pm 0.5 \mathrm{~K}$. PEHA0 and UPyPEHA2 are liquid-like at room temperature, and the resulting force is too small to be measured by the transducer at the relevant extensional rates. In contrast, the more highly cross-linked nature of UPyPEHA9 and UPyPEHA14 polymers meant that these could not be attached to the plate even at $T=403 \mathrm{~K}$; thus, only the UPyPEHA6 polymer was successfully measured using extensional rheology.

\section{RESULTS AND DISCUSSIONS}

\section{A. Linear viscoelasticity and the validity of TTS}

The SAOS results for our series of polymers were determined as outlined in the Experimental section. To obtain the SAOS response over a wider frequency range than what is possible in a single measurement, we investigate in detail to what extent TTS can be used to extend the dynamic range. We plot the loss tangent $\tan (\delta)$ as a function of the absolute value of the complex modulus $\left|G^{*}\right|$ in a VGP plot [96,97], Fig. 2. In a $\tan (\delta)$ vs $\left|G^{*}\right|$ representation, the SAOS data for PEHA0 and UPyPEHA2 [blue rings in Figs. 2(a) and 2(b)] are relatively smooth and continuous across the whole temperature range, indicating (but not guaranteeing) that TTS has the potential to work well for these two samples. However, for higher $\phi_{\mathrm{UPy}}$ and particularly for 9 and $14 \mathrm{~mol}$. $\%$, the curves (blue rings) show discontinuities from one temperature to the next in the mid-to-high modulus range. This behavior clearly indicates a failure of TTS at low temperatures. For each polymer, we estimate the temperature where the curves start to show clear discontinuities. Based on this information, we modify the master curve plots in Fig. 3(a) so that the TTS master curves are terminated at low temperatures, where we have indications that TTS is not a good approximation, and the shift factor plots resulting from this procedure are shown in Fig. 3(b), respectively. To only include the data for which we find strong indications of TTS working well (as we do above) is probably the most defensible position to take when TTS is found to break over some parts of the dynamic range. Certainly, we would expect TTS errors to be cumulative, such that a small TTS error repeated over many increments in temperature will add together to give a largely incorrect placement (and shape) of the data at temperatures distant from the reference (and correspondingly at frequencies distant from the measured frequency). Nevertheless, the question remains: relatively close to the original measurement frequency, how well do the TTS-shifted data actually represent the real behavior?

To further investigate the effect of the supramolecular interactions on TTS, and to test the accuracy of the TTS-shifted data, we compare these with the dynamic modulus data obtained from stress relaxation after step strain measurements performed on the same polymers, where the analysis software iRheo was used to perform the transformation from the stress relaxation data to the dynamic modulus. To test the reliability of the iRheo transformation, we take our stress relaxation result for UPyPEHA14 at $T=263 \mathrm{~K}$ as an example, and compare it with our TTS results. The results of this comparison are plotted in Fig. 4(a) (black lines from $i R h e o$ and green symbols from TTS). As expected, the moduli from the iRheo analysis and from the TTS analysis overlap well in the frequency range of a single SAOS 

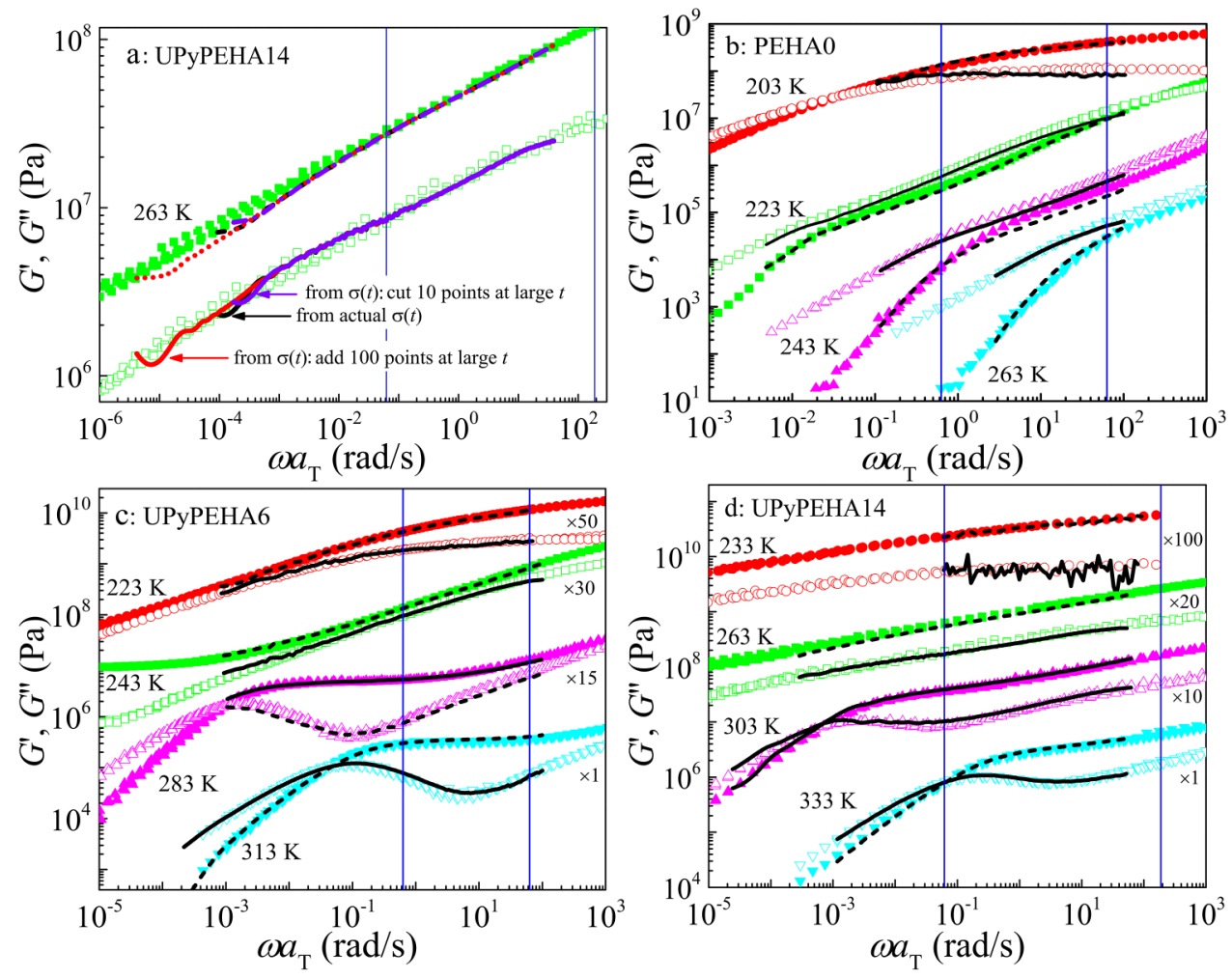

FIG. 4. Comparisons between the complex shear moduli obtained from TTS (symbols) and from the conversion of stress relaxation data using iRheo (lines); for the stress relaxation data, the shift parameter is $a_{T}=1$ (a) Data for UPyPEHA14 at $T=263 \mathrm{~K}$ which illustrate the accuracy of the $i R h e o$ conversion at low frequencies, as further described in the text. Data for several temperatures are shown for PEHA0 in (b), for UPyPEHA6 in (c), and for UPyPEHA14 in (d). The data for UPyPEHA6 and UPyPEHA14 in panels (c) and (d) are vertically shifted for clarity using the multiplication factors shown in the panels. The blue vertical lines indicate the frequency range of a single SAOS measurement.

measurement (between two vertical blue lines). However, since the stress for this sample does not fully relax in the time window of the $\sigma(t)$ step strain experiment, the iRheo transformation gives unphysical shapes in the low frequency range of its output. iRheo allows users to fit and extrapolate the $\sigma(t)$ curves at long times, which can improve the transformation at low frequencies [98]. To test to what degree we can trust the transformation result in the low frequency range, we altered the input $\sigma(t)$ data in two simple ways, and examined the transformed output. Firstly, we fit $\sigma(t)$ up to a time near the experimental end-time, and artificially extrapolate to longer time (equivalent to longer experiment time) to evaluate the effects of extending the dynamic range. Secondly, for further comparison, we investigate the effects of slightly decreasing the dynamic range by removing a few data points from the original $\sigma(t)$ curve near the experiment end-time. The transformation results obtained from the three $\sigma(t)$ curves are compared as the red, black, and purple lines in Fig. 4(a). It is clear from this comparison that the majority of the output is stable with respect to these changes in the input data, but the lowest frequency results (where the unphysical shapes are seen) are altered, as might be expected. We conclude that the transformation is uncertain in this low frequency regime, and thus cut the transformed output below the frequency where the three curves diverge. A similar procedure was followed for all other iRheo converted data reported in this paper.

We next compare the dynamic moduli obtained from TTS and stress relaxation for the three samples PEHA0,
UPyPEHA6, and UPyPEHA14 at a range of different temperatures, as shown in Figs. 4(b)-4(d); the symbols show the TTS results and the lines show the modulus converted from the stress relaxation experiments. The data at different temperatures for UPyPEHA6 and UPyPEHA14 in panels (c) and (d) are vertically shifted for clarity, using shift factors shown in the figure. It is worth noting that the $\sigma(t)$ curves for PEHA0 at $203 \mathrm{~K}$ and UPyPEHA14 at $233 \mathrm{~K}$ are somewhat noisy; thus, more points on the transformed modulus curves are cut. From the comparison in Figs. 4(b)-4(d), it is clear that the TTS curves (symbols) and iRheo results overlap reasonably well with each other in the extended frequency range covered by the iRheo output. This is perhaps surprising since, in at least some cases such as the UPyPEHA14 sample at $263 \mathrm{~K}$, the data span regions where TTS obviously breaks down (i.e., perfect overlap is not achieved in the TTS curves or in the VGP plots in Fig. 2). Nevertheless, the TTS shifted data do (on average) closely follow the overall shape of the $i R h e o$ output. One reason for this becomes evident on examining Fig. 2, where the stress relaxation results are also represented in the VGP plots for each sample [Figs. 2(a), 2(c), and 2(e)]. Where TTS is found to work for the oscillatory shear data (e.g., PEHA0 and much of the UPyPEHA6 data), the stress relaxation results follow the same curve as the oscillatory data, but span a wider range of moduli at each temperature. However, where TTS is breaking down (e.g., the UPyPEHA14 sample at $263 \mathrm{~K}$ ), the extended curve obtained by stress relaxation experiments at a given 


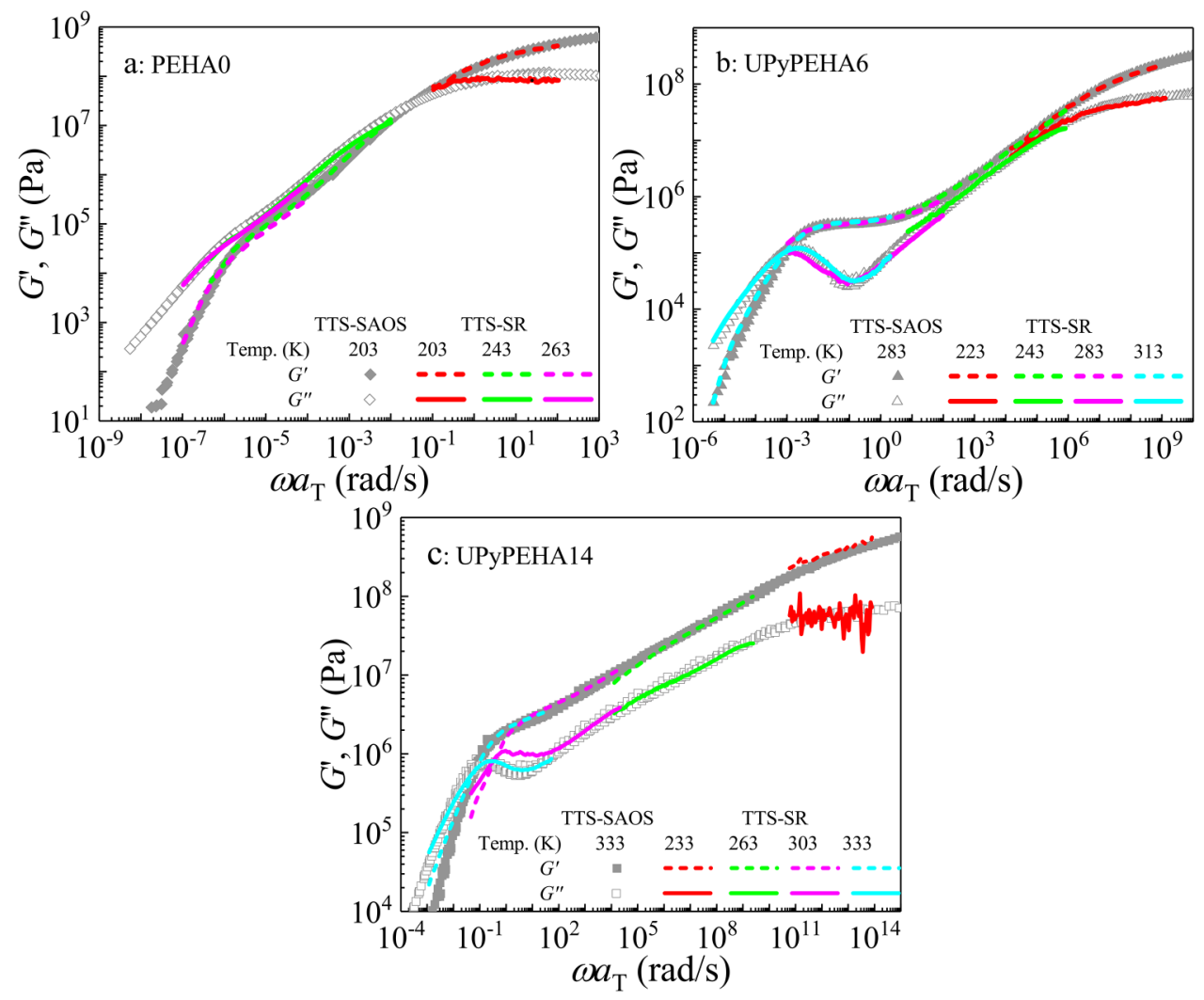

FIG. 5. A comparison of the master curves determined using TTS on SAOS data, including the full range of data (symbols), and from stress relaxation measurements (lines) shifted using the same TTS parameters determined from SAOS for (a) PEHA0, (b) UPyPEHA6, and (c) UPyPEHA14. The stress relaxation data shown in colored lines are shifted horizontally using the shift factors used to construct the TTS master curves at the corresponding temperatures. For example, the master curve for PEHA0 is constructed using a reference temperature of $T=203 \mathrm{~K}$. Thus, the red lines that refer to a temperature of $T=203 \mathrm{~K}$ are not shifted, but the green and pink lines are shifted using their corresponding TTS shift factors for $T=243$ and $263 \mathrm{~K}$, respectively.

temperature still passes through the broad band swept out by the (nonoverlapping) oscillatory rheology data taken at nearby temperatures, following the general shape of that band. The net result is that the cumulative error produced when TTS shifting oscillatory data obtained at temperatures close to the reference, are small. Consequently, the TTS curves match quite closely the iRheo output, over the frequency range obtainable by transforming stress relaxation data taken over a reasonable experimental time, as is clear in Fig. 4.

The error in TTS shifting, however, accumulates when data from a much broader range of temperatures is shifted by larger extents in the frequency domain. This is apparent in Fig. 5, where the stress relaxation data taken at different temperatures are shifted by the same factors needed to create "master curves" from the oscillatory data. We have here included the full range of TTS shifted oscillatory rheology data for comparison, thus not including only the data shown in 3(a), where TTS works well. Although the shifted stress relaxation data overlap with the shifted oscillatory data taken at the same temperature, there is evidently a mismatch between the shifted stress relaxation data obtained at different temperatures for the UPyPEHA14 sample (and weakly for the UPyPEHA6 sample). The PEHA0 data overlaps perfectly.

Hence, we conclude that construction of a reliable master curve across a broad frequency range is not possible for the samples with high $\phi_{\mathrm{UPy}}$; the cumulative shifting error means that sections of the spectrum are moved to the incorrect frequencies. In what follows, we thus use only the master curves depicted in Fig. 3(a) and the shift factors in Fig. 3(b), which contains only the data for which TTS are a reasonable approximation. The master curves obtained using TTS at a reference temperature of $363 \mathrm{~K}$ are shown in Fig. 3(a). For each polymer, the data taken at the reference temperature are shown in yellow and black lines for $G^{\prime}$ and $G^{\prime \prime}$, respectively, to allow for easy comparisons between the different polymers. The temperature-dependent horizontal frequency shift factors $a_{T}$ used to create the master curves are shown in Fig. 3(b).

TABLE II. WLF and Arrhenius fitting parameters for the LVE shift factors. For PEHA0 and UPyPEHA2, WLF fits were performed over the whole temperature range, whereas for the samples with $\phi_{\mathrm{UPy}} \geq 6 \mathrm{~mol} . \%$, Arrhenius fits were performed for temperatures above $T=323 \mathrm{~K}$.

\begin{tabular}{|c|c|c|c|c|}
\hline \multirow{2}{*}{ Sample codes } & \multicolumn{2}{|c|}{ WLF fits } & \multicolumn{2}{|c|}{ Arrhenius fits } \\
\hline & $C_{1}$ & $C_{2}$ & $-\log _{10}\left(a_{T}^{0}\right)$ & $\begin{array}{c}E_{\mathrm{a}} \\
(\mathrm{kJ} / \mathrm{mol})\end{array}$ \\
\hline PEHA0 & $9.9 \pm 0.3$ & $109.9 \pm 1$ & - & - \\
\hline UPyPEHA2 & $11.3 \pm 0.4$ & $89.9 \pm 0.9$ & - & - \\
\hline UPУРEHA6 & - & - & $19 \pm 0.2$ & $108 \pm 1.1$ \\
\hline UPУРЕНА9 & - & - & $22 \pm 0.1$ & $116 \pm 1.2$ \\
\hline UPyPEHA14 & - & - & $37 \pm 0.3$ & $191 \pm 0.6$ \\
\hline
\end{tabular}


From Fig. 3(a), the following general statements can be made: as $\phi_{\text {UPy }}$ increases, (i) the plateau moduli increase, (ii) the terminal relaxation times increase, and (iii) the power law exponents at the lowest measured frequencies decrease. These results are consistent with results for several other reported supramolecular polymers [63-66]. As discussed in the Introduction, we predict that all our polymer samples are unentangled. Thus, no plateau should be observed in the absence of supramolecular effects and this is indeed observed for the PEHA0 samples. Also the lowest UPy concentration sample UPyPEHA2 shows little evidence of a plateau. For the UPyPEHA samples containing more than 2 mol. \% UPy, however, we clearly observe rubber-like plateaus, and the plateau modulus increases systematically with increasing $\phi_{\mathrm{UPy}}$. This is expected since, as discussed above, the UPy dimers act as physical cross-links leading to the formation of an elastic network. Moreover, it is also clear that addition of associating UPy groups leads to a delay of the terminal relaxation for all supramolecular polymers compared to the nonsupramolecular polymer PEHA0 and that the terminal relaxation times increase with increasing $\phi_{\mathrm{UPy}}$. The temperature-dependent shift factors for PEHA0 and UPyPEHA2 can be well described using a WLF expression, $\log \left(a_{T}\right)=\frac{-C_{1}\left(T-T_{\text {ref }}\right)}{C_{2}+\left(T-T_{\text {ref }}\right)}$, where $a_{T}$ is the temperature-dependent shift-factor, $T_{\text {ref }}=363 \mathrm{~K}$ is the reference temperature and $\mathrm{C}_{1}$ and $\mathrm{C}_{2}$ are constants, as shown in Table II. In the temperature range where we find that TTS works well (above $T=323 \mathrm{~K}$ ) for UPyPEHA with $\phi_{\mathrm{UPy}} \geq 2 \mathrm{~mol}$. $\%$, the shift factors can be fitted using an Arrhenius expression, $a_{T}=a_{T}^{0} \exp \left(E_{\mathrm{a}} / k_{\mathrm{B}} T\right)$, where $a_{T}^{0}$ are prefactors and $E_{\mathrm{a}}$ denotes the activation energies for polymers to flow (see Table II); we find that the activation energies increase as $\phi_{\mathrm{UPy}}$ increases.

\section{MODELING OF LINEAR SHEAR AND NONLINEAR EXTENSIONAL RHEOLOGY}

\section{A. "Classic" sticky-Rouse model}

The most commonly used model to describe the rheology of unentangled associating polymers, the sticky-Rouse model, is based on the idea that stickers along the chain provide an additional effective drag, delaying the terminal relaxation time [41,80-82]. The chemical dissociations and associations of the stickers occur on a time scale $\tau_{\text {assoc }}$, corresponding to the typical time a sticker will spend associated. However, following the idea of Rubinstein and Semenov [82], a dissociated sticker will often return to and reassociate with the same partner. Hence, a significant stress relaxation only occurs when the stickers change partners, characterized by an average timescale $\tau_{\mathrm{s}}$, which may be significantly longer than the timescale $\tau_{\text {assoc. }}$. Thus, $\tau_{\mathrm{s}}$ is the relevant timescale for linear rheology. We assume that the sticker lifetime $\tau_{\mathrm{s}}$ is significantly longer than the timescale for the slowest Rouse-mode corresponding to chain segments between stickers, and thus $\tau_{\mathrm{s}} \gg(N / \mathrm{S})^{2} \tau_{0}$, where $\tau_{0}$ is the characteristic relaxation time of a Rouse monomer, $\mathrm{S}=M / M_{\text {strand }}$ is the average number of stickers per chain of molecular mass $M$, $M_{\text {strand }}$ is the average molar mass between stickers, and $N$ is the degree of polymerization of the chain.
We note that for gel-forming associating polymers, Zhang et al. [100] have suggested a simple relationship between the Rouse monomer time $\tau_{0}$ and the association time $\tau_{\text {assoc }}$ via the activation energy characterizing flow, $E_{\mathrm{a}}$ $\left(\tau_{\text {assoc }}=\tau_{0} \exp \left(E_{\mathrm{a}} / k_{\mathrm{B}} T\right)\right.$ ), noting, in particular, that $\tau_{0}$ itself is temperature-dependent. This relationship assumes that the Rouse physics applies to the local environment of the sticker groups and was demonstrated to describe gel-forming polymer systems with relatively few stickers per chain. For our polymers, however, the terminal stress relaxation and flow is controlled by the time-scale $\tau_{\mathrm{s}}$, which cannot be simply linked to $\tau_{\text {assoc }}$, and we thus do not find this approach applicable here.

The slowest Rouse modes are uniformly retarded by the effective sticker friction, and so it is possible to decouple the stress relaxation function, $G(\mathrm{t})$, into two distinct summations over mode contributions, as proposed by Chen and co-workers [41]. The first term in Eq. (1) is the contribution to $G(t)$ from chain strands longer than $M_{\text {strand }}$ that are unrelaxed, and thus elastically active at time $t$, and the second sum is the corresponding Rouse contribution from chain strands shorter than $M_{\text {strand }}$

$$
\begin{aligned}
G(t)= & \sum_{i} w_{i} \frac{\rho R T}{M_{i}}\left[\sum_{p=1}^{\mathrm{S}_{i}} \exp \left(-t p^{2} / \tau_{\mathrm{s}} \mathrm{S}_{i}^{2}\right)\right. \\
& \left.+\sum_{p=\mathrm{S}_{i}+1}^{N_{i}} \exp \left(-t p^{2} / \tau_{0} N_{i}^{2}\right)\right] .
\end{aligned}
$$

Here, $\rho$ is the mass density of the polymer, $R$ the ideal gas constant, $T$ the temperature, $w_{i}$ and $M_{i}$ are the weight fraction and molecular weight of the $i$ th chain fraction, $N_{i}=M_{i} / M_{0}$ is the number of elementary Rouse monomers per chain, each with molar mass $M_{0}$, and $\mathrm{S}_{i}$ is the average number of stickers on the $i$ th chain fraction. Note that we have the relation

$$
M_{\text {strand }}=\rho R T / G_{N}^{0}
$$

where $G_{N}^{0}$ is the (experimental) value of the plateau modulus. Given that $\rho, T$, and $M_{i}$ are known, $G_{N}^{0}, \tau_{\mathrm{s}}$, and $\tau_{0}$ are fitting parameters of this model, where the two timescale parameters shift the model predictions in time, or correspondingly frequency, in a frequency-dependent representation.

In this work, we demonstrate that the sticky-Rouse model can capture the low frequency, long-time, linear rheological response for all four polymers. However, the model fails to predict the loss modulus at intermediate frequencies around the plateau region $[41,65]$. Hence, we propose a number of modifications of the sticky-Rouse model based on physical arguments aimed to improve the mid-frequency predictions and to be able to assess the relevance of fitted parameters.

Firstly, we note that the synthesis process, random copolymerization, leads to a random placement of the stickers along the backbone and this is not accounted for in Eq. (1), which assumes that all stickers are equally spaced. Secondly, in Eq. (1), the relaxation of chain-end segments (one free end and one associated) is treated in the same way as the chain segments "trapped" between stickers (both associated). Thus, we shall differentiate between these two "types" of chain 
segments. Accounting for these two factors leads to a modification of the "fast" relaxation modes of the sticky-Rouse spectrum.

At the time scale of $\tau_{\mathrm{s}}$, or longer, only the "trapped" chain segments contribute to the stress because the chain ends and internal modes of the trapped chains are fully relaxed. A model thus needs to be consistent with the random sticker placement and be able to describe the relaxation of the remaining chain modes. It would be possible to treat the long time motion of a chain by constructing a Rouse-like model with a friction proportional to $\tau_{\mathrm{s}}$ concentrated at the randomly placed sticker positions. However, this would not properly represent the chain motion, since dissociation of a sticker and reassociation with a new group involves a finite sized "hop" of the chain, with a hop amplitude dependent upon the lengths of chain to adjacent stickers, rather than a continuous motion with increased friction. Thus, we instead construct, below, a stochastic model with finite sized hops. This part of our model shares some features with the model described earlier by Shivokhin et al. [76] for entangled sticky polymers, and may be considered a special case of that model.

\section{B. Placement of stickers on a chain}

We first generate a numerical ensemble of chains that accounts for the distribution of the distance between stickers and the length of the dangling ends.

For a given molecular mass, $M$, we build $\mathrm{C}$ chains. Beginning from one chain end, we generate a series of molecular masses, $M_{i}$, which defines the distance to consecutive stickers, from the probability distribution [7,101]:

$$
p\left(M_{i}\right)=\frac{1}{M_{\text {strand }}} \exp \left(-\frac{M_{i}}{M_{\text {strand }}}\right) .
$$

This equation assumes that during chain polymerization, sticker groups are added to the chain in a purely random fashion. Hence, starting from any point on the chain, the probability distribution for the distance to the next sticker will follow the above exponential distribution, and the total number of stickers on chains of a given molecular weight correspondingly follows a Poisson distribution.

We add the first sticker at a distance $M_{1}$ from the chain end, and then generate a new $M_{i}$ for the distance to the next sticker, and so on. Hence, the first sticker is placed after a chain length $M_{1}$, then another sticker is placed after a chain length $M_{2}$, etc., until we exceed the given molecular weight of the considered chain, i.e., we stop when $\sum_{i} M_{i}>M$. A

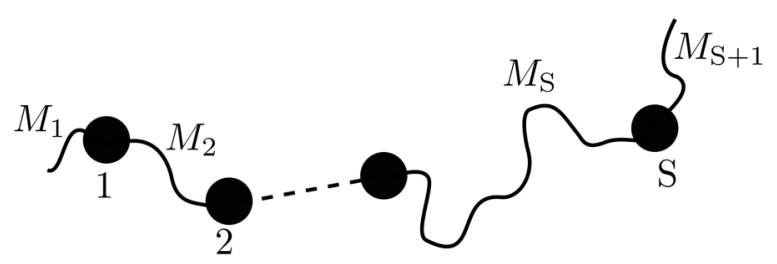

FIG. 6. Cartoon of the system. $\mathrm{S}$ stickers are randomly placed along the backbone, separated by chain strands of molar masses $M_{i} . M_{1}$ and $M_{\mathrm{S}+1}$ are the molar masses of the two chain-ends. typical chain resulting from this procedure is shown in Fig. 6. We generate $\mathrm{C}$ chains according to this process, which typically results in a set of chains as presented in Fig. 7. Each chain, $k$, has $\mathrm{S}_{k}$ stickers distributed along the chain according to the set of strand molar masses connecting them: $\left\{M_{k, i}\right\}, i=\left\{1,2, \ldots, \mathrm{S}_{k}\right\}$. From this process, we obtain chains with a distribution of distances between stickers and (as noted above) a Poisson distribution for the number of stickers per chain. Since the average chain strand molecular mass between stickers, $M_{\text {strand }}$ is independent of the chain molecular mass, see Eq. (2), the average number of stickers per chain increases with increasing chain molecular mass.

In Sec. IV C, we detail how the stress relaxation function is computed for a set of chains as generated above.

\section{Stress relaxation in the stochastic sticky-Rouse model}

As discussed in Sec. IV B, we decouple, similarly to Eq. (1), the contribution of the "fast" Rouse modes and the "slow" sticky modes to the total stress relaxation, $G_{\text {stocha, and }}$ write

$$
G_{\text {stocha }}(t)=G_{\text {fast }}(t)+G_{\text {sticky }}(t) \text {. }
$$

We defer the technical details of the calculations of $G_{\text {fast }}$ and $G_{\text {sticky }}$ to Appendix and summarize their expressions in what follows.

\section{Fast Rouse relaxation modes}

The "fast" relaxation modes-on a timescale where the sticker configurations do not change-are decomposed into two contributions from (i) the dangling chain ends and (ii) all the other chain strands (trapped between two stickers). We have

$$
G_{\text {fast }}(t)=\sum_{\ell=1}^{q} w_{\ell} \frac{\rho R T}{M_{\ell}}\left\{\tilde{G}_{\text {ends }, \ell}(t)+\tilde{G}_{\text {trapped }, \ell}(t)\right\}
$$

where we have considered polydispersity by discretizing the molecular weight distribution into $q$ modes (of weight $w_{\ell}$ and molecular weight $M_{\ell}$ for each mode $\ell$ ), and where the

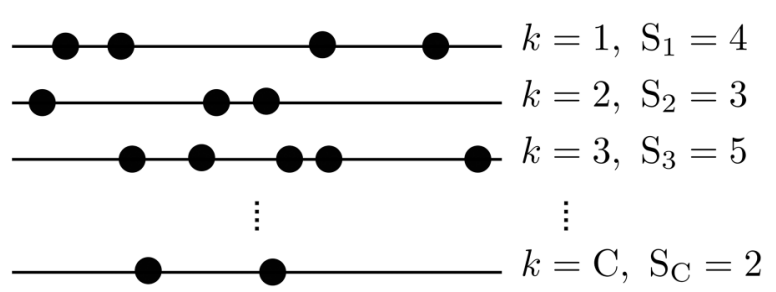

FIG. 7. Example of a set of $\mathrm{C}$ chains. On each chain, $k$, the stickers (black circles) are placed via Eq. (3). 
(tilded) dimensionless stress relaxation functions are

$$
\tilde{G}_{\text {ends }, \ell}(t)=\frac{1}{\mathrm{C}_{\ell}} \sum_{k=1}^{\mathrm{C}_{\ell}} \sum_{i=\left\{1, \mathrm{~S}_{\ell, k}+1\right\}} \sum_{p=1, p_{\text {odd }}}^{N_{\ell, k, i}} \exp \left(-\frac{t p^{2}}{4 N_{\ell, k, i}^{2} \tau_{0}}\right)
$$

$$
\tilde{G}_{\text {trapped }, \ell}(t)=\frac{1}{\mathrm{C}_{\ell}} \sum_{k=1}^{\mathrm{C}_{\ell}} \sum_{i=2}^{\mathrm{S}_{\ell, k}} \sum_{p=1}^{N_{\ell, k, i}} \exp \left(-\frac{t p^{2}}{N_{\ell, k, i}^{2} \tau_{0}}\right)
$$

where

- $\mathrm{C}_{\ell}$ is the number of simulated chains of molecular weight $M_{\ell}$,

- $\mathrm{S}_{\ell, k}$ is the number of stickers on the chain $k$ of molecular weight $\ell$,

- $N_{\ell, k, i}$ is the number of elementary segments on the strand $i$ of chain $k$ of molecular weight $\ell$, and

- $\tau_{0}$ is the relaxation time of an elementary chain segment.

These stress relaxation functions are essentially Rouse relaxations decorated to account for each chain's random sticker placement produced according to Sec. IV B.

\section{Slow sticky relaxation modes}

In contrast with the above "fast" stress relaxation, the "slow" stress relaxation-on a timescale where the dangling chain ends and strands of chains between sticker have relaxed-is calculated by allowing stickers to take "hops," i.e., stickers detach and reattach in a different spatial location, as shown in Fig. 8. The place where the sticker $i$ reattaches is the weighted average position, $\bar{R}_{i}$, which is determined by its two neighboring stickers, plus a random displacement around that position, $\Delta R_{i}$, drawn from a Gaussian probability distribution whose variance, $\sigma_{i}^{2}$, depends on the "size" of the two chain strands the sticker $i$ is connected to:

$$
\sigma_{i}^{2}=\frac{k_{\mathrm{B}} T}{k_{\mathrm{eff}, i}} \quad \text { with } \quad k_{\mathrm{eff}, i}=\frac{3 k_{\mathrm{B}} T}{b^{2} N_{i}}+\frac{3 k_{\mathrm{B}} T}{b^{2} N_{i+1}},
$$

where $N_{i}, N_{i+1}$ are the number of elementary chain segments (each of length $b$ ) in the chain strands connected to the sticker $i$.

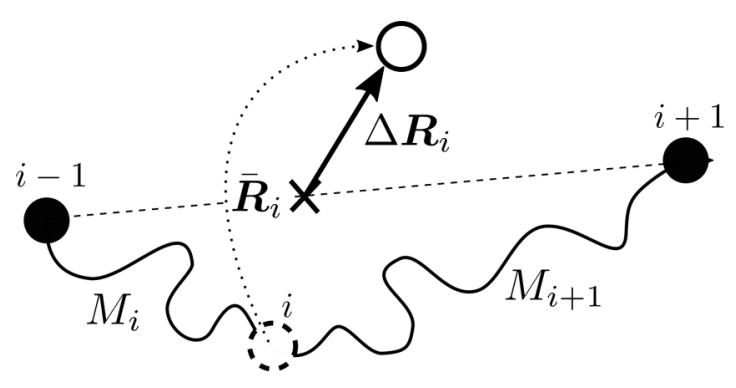

FIG. 8. Sticker $i$ detaches (dashed circle), takes a local "hop," and reattaches to a new position: $R_{i}^{\text {new }}=\bar{R}_{i}+\Delta R_{i}$, (empty circle).
We allow many subsequent sticker "hops," by each time picking a sticker at random amongst all the stickers on the $\mathrm{C}_{\ell}$ chains and placing it at a new position $R_{i}=\bar{R}_{i}+\Delta R_{i}$.

We record the fluctuations in the stress tensor for a stochastic simulation of the hopping chains with stickers, run at equilibrium over a long period of time. The stress relaxation function is obtained from the stress fluctuations by means of the fluctuation-dissipation theorem [102,103]. Considering polydispersity we have

$$
G_{\text {sticky }}(t)=\sum_{\ell=1}^{q} w_{\ell} \frac{\rho R T}{M_{\ell}} \tilde{G}_{\text {sticky }, \ell}(t),
$$

where the dimensionless stress relaxation function for each component $\ell$ of the molecular weight distribution is defined as

$$
\begin{gathered}
\tilde{G}_{\text {sticky }, \ell}(t)=\frac{1}{\mathrm{C}_{\ell}}\left\langle\tilde{\sigma}_{x y, \ell}(t+\tau) \tilde{\sigma}_{x y, \ell}(\tau)\right\rangle \text { with } \\
\tilde{\sigma}_{x y, \ell}=\sum_{k=1}^{\mathrm{C}_{\ell}} \sum_{i=2}^{\mathrm{S}_{\ell, k}} \frac{3}{b^{2} N_{i}} R_{\ell, k, i, x} R_{\ell, k, i, y} .
\end{gathered}
$$

Note that averaging over different directions, as shown in the Appendix, improves the statistical accuracy of $\tilde{G}_{\text {sticky }, \ell}$.

\section{Sticker times}

In this section, we will show that, to compare the values of the sticker time $\tau_{\mathrm{s}}$ in the "classic" sticky-Rouse model [Eq. (1)] with the stochastic sticky-Rouse model in a fair way, we need to multiply the former by a factor $\pi^{2}$. To do so, we take the special case where the stickers are equally spaced along the chain. Therefore, the number of Rouse monomers between stickers is fixed to $N_{\mathrm{m}}=N / \mathrm{S}$, and so Eq. (8) reduces to

$$
k_{\mathrm{eff}}=\frac{3 k_{\mathrm{B}} T}{b^{2} N_{\mathrm{m}}}+\frac{3 k_{\mathrm{B}} T}{b^{2} N_{\mathrm{m}}}=\frac{6 k_{\mathrm{B}} T}{b^{2} N_{\mathrm{m}}} .
$$

In Eq. (8), $\sigma_{i}^{2}$ represents the mean square displacement around the mean position defined by $\bar{R}$. Figure 9 illustrates this process, projected on the $x$-axis. Upon detachment, a sticker "hops" to its new position defined as

$$
x^{\text {new }}=\bar{x}_{i}+\sigma_{i} \text {. }
$$

This new position is, on average, at a distance $\sqrt{2} \sigma_{i}$ away from its current position (because $\sigma_{i}$ is measured from the center position $\bar{x}_{i}$, and we add the variance). Therefore, the

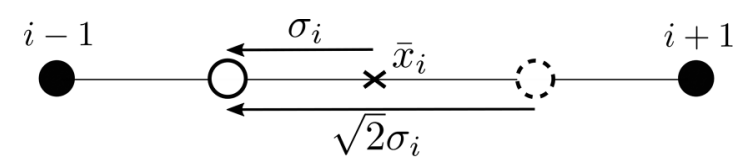

FIG. 9. Sticker "hop" projected on the $x$-axis. Upon detachment, its new position (empty circle) is defined, on average, as $x^{\text {new }}=\bar{x}_{i}+\sigma_{i}$, which is, on average, $\sqrt{2} \sigma_{i}$ away from its current position (dashed circle). 
actual mean square displacement of the sticker $\left\langle\Delta x^{2}\right\rangle$, is

$$
\begin{aligned}
\left\langle\Delta x^{2}\right\rangle & =2 \sigma_{i}^{2} \\
& =2 k_{\mathrm{B}} T / k_{\mathrm{eff}} \\
& =\frac{b^{2} N_{\mathrm{m}}}{3} .
\end{aligned}
$$

In one dimension, the effective diffusion coefficient, $D$, is of the form

$$
\left\langle\Delta x^{2}\right\rangle=2 D t
$$

where $t \equiv \tau_{\mathrm{s}}$. Hence, we have

$$
D=\frac{b^{2} N_{\mathrm{m}}}{6 \tau_{\mathrm{s}}}
$$

and we can define the effective sticker friction coefficient as

$$
\begin{aligned}
\zeta_{\text {sticker }} & \equiv k_{\mathrm{B}} T / D \\
& =\frac{6 \tau_{\mathrm{s}} k_{\mathrm{B}} T}{b^{2} N_{\mathrm{m}}} .
\end{aligned}
$$

Now, we can use the definition of the Rouse time for a chain of $N$ beads, of friction coefficient $\zeta$, connected by springs of length $b$ [104]:

$$
\tau_{\mathrm{R}}=\frac{\zeta N^{2} b^{2}}{3 \pi^{2} k_{\mathrm{B}} T}
$$

To find the Rouse time of a chain composed of S "springs" of step length $\left(N_{\mathrm{m}} b^{2}\right)^{1 / 2}$, we, therefore, make the following substitutions in Eq. (17):

\begin{tabular}{|c|c|c|c|c|c|c|c|}
\hline \multirow{2}{*}{$\begin{array}{l}\text { Sample } \\
\text { codes }\end{array}$} & \multirow{2}{*}{$\begin{array}{c}M_{\mathrm{n}}{ }^{\mathrm{a}} \\
(\mathrm{kg} / \\
\mathrm{mol})\end{array}$} & \multirow{2}{*}{$\mathrm{PDI}^{\mathrm{a}}$} & \multicolumn{2}{|c|}{ S } & \multicolumn{2}{|c|}{$\tau_{\mathrm{s}}(\mu \mathrm{s})$} & \multirow{2}{*}{$\begin{array}{c}\tau_{0} \\
\text { (ns) }\end{array}$} \\
\hline & & & $\mathrm{NMR}^{\mathrm{a}}$ & Models $^{\mathrm{b}}$ & Stochastic & Classic $^{c}$ & \\
\hline UPyPEHA2 & 16.6 & 1.24 & 2 & 0.2 & 5 & 25 & 0.70 \\
\hline UPyPEHA6 & 22.0 & 1.38 & 7 & 3 & 29 & 65 & 1.0 \\
\hline UPyРEHA9 & 23.7 & 1.71 & 11 & 8 & 56 & 100 & 2.5 \\
\hline UPyPEHA14 & 24.6 & 2.38 & 17 & 21 & 83 & 200 & 500 \\
\hline
\end{tabular}

$$
N \rightarrow \mathrm{S}, \quad b^{2} \rightarrow N_{\mathrm{m}} b^{2}, \quad \zeta \rightarrow \zeta_{\text {sticker }}
$$

to obtain the Rouse relaxation time of a Rouse chain composed of $\mathrm{S}$ springs

$$
\begin{aligned}
\tau_{\mathrm{R}} & =\frac{\zeta_{\text {sticker }} \mathrm{S}^{2} N_{\mathrm{m}} b^{2}}{3 \pi^{2} k_{\mathrm{B}} T} \\
& =\frac{2 \mathrm{~S}^{2} \tau_{\mathrm{s}}}{\pi^{2}}
\end{aligned}
$$

TABLE III. Parameters used in the stochastic and "classic" sticky-Rouse models.

${ }^{\text {a }}$ Identical to values of Table I.

${ }^{\mathrm{b}}$ Same parameters used in both models.

${ }^{\mathrm{c}}$ Includes the factor $\pi^{2}$, Sec. IV C 3 .
Finally, the relaxation modulus for such chain is

$$
G(t)=\frac{\rho R T}{M} \sum_{p} \exp \left(\frac{-2 p^{2} t}{\tau_{\mathrm{R}}}\right) .
$$

The reason for the factor of two appearing in the exponential is that there is a factor of two difference between the relaxation time for the stress contribution of the $p$ th mode and the relaxation time of molecular orientation from the $p$ th mode $\left(\tau_{\mathrm{R}}\right)$ [105]. Using Eq. (18), we obtain

$$
G(t)=\frac{\rho R T}{M} \sum_{p} \exp \left(\frac{-\pi^{2} p^{2} t}{\mathrm{~S}^{2} \tau_{\mathrm{s}}}\right) .
$$

Comparing the latter expression for the relaxation modulus with the corresponding term in Eq. (1), we see that there is a factor $\pi^{2}$ difference. Therefore, if we want to compare the sticker-time parameter of the stochastic sticky-Rouse model with the sticker-time parameter of the "classic" sticky-Rouse model, Eq. (1), we need to multiply the latter by a factor $\pi^{2}$. This factor $\pi^{2}$ is included in the value reported in Table III.

\section{Comparison with experimental data}

\section{Fit to the data}

Figures 10(a) and 10(b) show storage and loss moduli of experimental data, as reported above in Fig. 3(c), together with fits to the data using the stochastic model and the sticky-Rouse model for the UPyPEHA6 and an indication of the contribution to the spectrum from fast internal Rouse modes and "sticky" modes.

Figure 10(a) shows the fitting results of both models for the sample with different UPy contents. The parameters used for both models are shown in Table III. The parameter S, which describes the average number of stickers per chain, fixes the value of the plateau modulus (of $G_{\text {sticky }}^{\prime}$ ) via Eq. (2). The parameter $\tau_{\mathrm{s}}$ effectively shifts $G_{\text {sticky }}^{\prime}$ and $G_{\text {sticky }}^{\prime \prime}$ horizontally and is adjusted to fit the low frequency cross-over. The parameter $\tau_{0}$ effectively shifts $G_{\text {fast }}^{\prime}$ and $G_{\text {fast }}^{\prime \prime}$ horizontally, and is adjusted to fit the high frequency region near the plateau modulus.

The slopes at low frequency are well captured both by the stochastic model and the sticky-Rouse model, which indicates that the "hop" picture described above for the large scale chain motion is meaningful, and that the polydispersity of the system is well captured in both models. However, the value of the relevant fitting parameter, the sticker time $\tau_{\mathrm{s}}$, is substantially different for the two models. The reason for this discrepancy is that the stochastic model considers finite distance hops of the discrete sticker groups, which are randomly placed along the chain, whereas the "sticky-Rouse model" of Eq. (1) considers continuous motion of a chain with distributed friction. We argue that the stochastic model is closer to the physical reality.

For frequencies in the rubbery plateau range, both models show a slight disagreement with the data, particularly in the loss modulus. For all materials, the fit is better for the stochastic model, because it includes: (i) the distribution of length between stickers and stickers per chains and (ii) the separate contribution of the chain ends. Both of these factors 

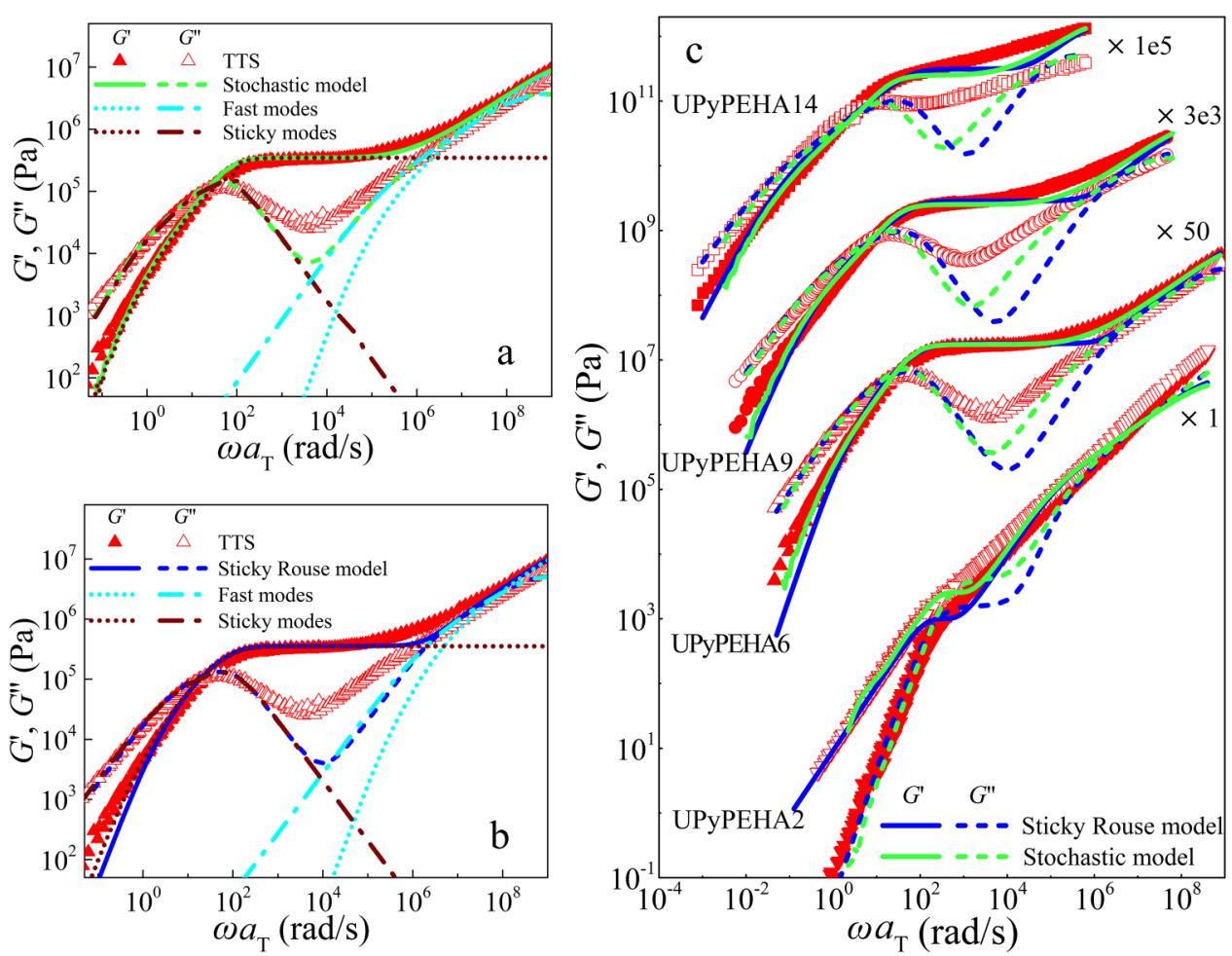

FIG. 10. TTS master curves (symbols) for UPyPEHA6 using a reference temperature of $T=343 \mathrm{~K}$ together with the fitting results (solid lines) using (a) the new stochastic model described in the text and (b) the sticky-Rouse model; the contributions from the sticky modes and the fast modes are also shown separately. (c) TTS master curves (symbols) for the samples with varying UPy content together with the results of the fitting using the stochastic model and the sticky-Rouse model, respectively. Note that the curves in (c) are vertically shifted for clarity using the multiplication factors shown in the figure. The fitting parameters used in the fits to both models are given in Table III.

give rise to a longer, smoother crossover between the high frequency Rouse spectrum (for sections of chain between stickers) and the plateau, because this crossover occurs at later times for longer chain sections between stickers, and for chains with one free end. Consequently, the storage and loss moduli are matched quite well at the high frequency end of the plateau region in the stochastic model, showing significant improvement over the sticky-Rouse model. Further improvement of the fit to data would require introducing yet more physical mechanisms with correspondingly more parameters. We consider a likely cause of the mismatch with data in the plateau region is that the model considers a single "sticker time.' Introducing a spectrum of sticker times (which might be justified by supposing different local environments or configurations for paired stickers) would certainly improve the model fits, even though this would come at the expense of introducing and parameterizing a function for the distribution of sticker lifetimes. A further possibility at the higher sticker concentrations is that some of the stress is held in torsional modes of the chains: this component of the stress can be relaxed even if a sticker returns to the same partner, i.e., at timescales related to $\tau_{\text {assoc }}$ rather than $\tau_{\mathrm{s}}$.

\section{2. $\tau_{\mathrm{s}}$ vs $\phi_{U P y}$}

One feature of the fitted value of the sticker time $\tau_{\mathrm{s}}$, that is true for both models, is that it increases with increasing concentration of stickers. It has been pointed out by Rubinstein and Semenov [82] that the effective sticker time in the model is not the fundamental time for sticker dissociation, since a dissociated sticker will return many times to the same partner, before finally finding another free partner with which to associate. Since returns to the same partner do not result in a significant chain rearrangement, to a first approximation, these events do not relax the stress. Consequently, the sticker time, $\tau_{\mathrm{s}}$, should be interpreted as the time to find a new partner. While it may be that the fundamental sticker dissociation time changes with UPy content (and certainly the activation energy does seem to change), it is appealing to suggest that the differences in sticker time $\tau_{\mathrm{s}}$ might be attributed to the relative difficulty of finding a new partner as UPy content is increased, i.e., stickers return more often to the same partner before swapping. This suggestion can be supported with a scaling argument showing that an increase of the number of UPy group per chain implies a decrease of the number of stickers present in a "search" volume of a detached sticker. Calling $b^{3}$ the volume of a sticker or monomer, and $N_{m} \equiv N / \mathrm{S}$ the average number of monomers between stickers, then the number of stickers per unit volume, $n_{\mathrm{s}}$, is defined as

$$
\begin{aligned}
n_{\mathrm{S}} & =\frac{\text { number of stickers per chain }}{\text { volume occupied by a chain }} \approx \frac{\mathrm{S}}{\mathrm{S} N_{m} b^{3}} \\
& =\left(N_{m} b^{3}\right)^{-1} .
\end{aligned}
$$

When a sticker is free, it explores its neighborhood in a volume limited by the typical dimensions of the chain between stickers, see Fig. 8, which obeys random walk 
statistics. Hence the explored volume, $V_{\text {search }}$, is of order

$$
V_{\text {search }} \approx\left(N_{m}^{1 / 2} b\right)^{3} \text {. }
$$

Therefore, the number of stickers, $N_{\text {partner }}$, in the exploration volume defined by Eq. (22) scales as

$$
\begin{aligned}
N_{\text {partner }} & \approx n_{\mathrm{s}} V_{\text {search }} \\
& \approx N_{m}^{1 / 2} \\
& \propto \mathrm{S}^{-1 / 2} .
\end{aligned}
$$

$N_{\text {partner }}$ represents the number of potential partners available to a sticker, and it decreases as the number of stickers per chain, S, increases. Consequently, we expect the effective sticker time $\tau_{\mathrm{s}}$ to increase with increasing number of stickers per chain. The above argument may be augmented by noting that for the largest concentration of stickers, the chain between stickers may in fact be not completely flexible. From Table I, we see that as $\phi_{\text {UPy }}$ increases, $N_{m}$ decreases from 16 for UPyPEHA6 to 6 for UPyPEHA14. Compared with the Kuhn length $(\sim 13$ monomers $)$ of a similar polymer (PHA) [95], the segments between two UPy groups are not flexible enough to search for a different partner in a surrounding volume, especially for high $\phi_{\mathrm{UPy}}$ samples. To relax stresses, cooperative dissociation of several stickers simultaneously is required for segments being flexible enough to search for a new partner. In other words, the activation energies for chain relaxations are determined by the event involving breaking of several stickers simultaneously. As $\phi_{\mathrm{UPy}}$ increases (segments between two UPy groups becomes shorter), more stickers are required to be broken simultaneously for chain relaxations, leading to higher activation energies. This argument is consistent with the results from Fig. 3(b), that activation energies for UPyPEHA2, UPyPEHA6, UPyPEHA9, and UPyPEHA14 are 78, 108, 116, and $191 \mathrm{~kJ} / \mathrm{mol}$, respectively.

\section{E. Extensional rheology and modeling}

To facilitate the design and to optimize the processing routes of materials based on supramolecular polymers, it is important to understand the material rheological response within the nonlinear regime. Although the linear viscoelasticity of supramolecular polymers are increasingly being investigated, there has been much less focus on the nonlinear rheology and particularly extensional rheology and fracture behavior. Here, as noted above in Sec. II, we were only able to successfully measure the nonlinear extensional rheology for the UPyPEHA6 sample. Figure 11(a) shows the extensional stress growth coefficient (i.e., stress divided by strain rate, $\sigma / \dot{\varepsilon}$ ) as a function of time for UPyPEHA6 with various stretching rates at $T=343 \mathrm{~K}$, while Fig. 11(b) shows the stress as a function of Hencky strain for the same measurements.

We find that at short times before strain hardening occurs, the tensile (extensional) stress growth coefficient, $\eta_{\mathrm{E}}^{+}$, data follow the LVE envelope [Eq. (26)]. However, for extension rates of $0.1 \mathrm{~s}^{-1}$ or below, at longer time (above a Hencky strain of 2), strain hardening is observed leading to tensile stress growth coefficients, $\eta_{\mathrm{E}}^{+}$, with magnitudes up to 10 times higher than those of the LVE envelope. For these cases, the experiments were terminated when stresses were out of the limit of the rheometer transducer. For Hencky strain rates $0.1 \mathrm{~s}^{-1}$ or above, the extension is typically terminated for stresses in the region of $(1-2) \times 10^{5} \mathrm{~Pa}$ (almost independent of strain rate) by brittle fracture. For the measurements performed at rates of $0.3 \mathrm{~s}^{-1}$ and above, the samples broke before strain hardening took place.

The solid lines in Figs. 11(a) and 11(b) correspond to the simplest possible nonlinear rheological description of the data, in which each mode from the multimode Maxwell fit of the LVE data is assigned to an upper-convected Maxwell model (UCM) [104]. The UCM model is one of the few nonlinear models that can be exactly derived from a microscopic model without closure approximations. A collections of dumbbells (frictional beads separated by linear springs) embedded in a fluid give rise to a UCM model with a single relaxation time. A "Rouse model" (many frictional beads with linear springs) in nonlinear flow gives rise to a superposition of multiple UCM models with relaxation times of these models corresponding to the Rouse relaxation spectrum [104]. Hence, if our polymer sample obeys Rouse-like dynamics, we may expect a multimode UCM model to successfully describe the data.

It is important to emphasize that the UCM model does not use "sticky" physics, which is in contrast with the main body of this paper, especially Sec. IV. It is reasonable to ask, therefore, why it is be possible to describe our data with the simple UCM model. The reason is that the extensional flow rates are extremely slow with respect to the sticker timescale. Indeed, it is possible to define a Weissenberg number with respect to the sticker time, $\mathrm{Wi}_{\mathrm{s}}=\dot{\varepsilon} \tau_{\mathrm{s}}$. Using the value of $\tau_{\mathrm{s}}$ from Table III, we see that for the flow rates used $\mathrm{Wi}_{\mathrm{s}}$ is at most $2 \times 10^{-5}$ (at the highest flow rate). Hence, during even a small amount of extensional strain, each sticker will have reconnected many times and chains will have reconfigured substantially. Under such circumstances, a coarse grained Rouse model with smooth friction is appropriate, and the details of the sticker dynamics are not important until large strains where chain forces become large. Thus, we can model the data without recourse to a detailed simulation of sticker dynamics. A similar conclusion may be arrived at by examining the linear rheology for the sample in Fig. 10(a), noting that the nonlinear extension rates used correspond to low frequencies towards the extreme left of the spectrum, i.e., at much lower frequencies than the inverse sticker time.

We test this as follows. We fit the LVE data by assigning two Maxwell modes per decade (via a least square procedure) to obtain a set of relaxation times, $\tau_{i}$, and weighting constants, $g_{i}$. The total stress is then given by

$$
\sigma(t)=\sum_{i} g_{i} A_{i}(t),
$$

where $A_{i}$ is the conformation tensor associated with the $i$ th Maxwell mode, whose time evolution is given by the upper convected Maxwell model

$$
\frac{\mathrm{d} A_{i}}{\mathrm{~d} t}=\kappa \cdot A_{i}+A_{i} \cdot \kappa^{T}-\frac{1}{\tau_{i}}\left(A_{i}-I\right),
$$



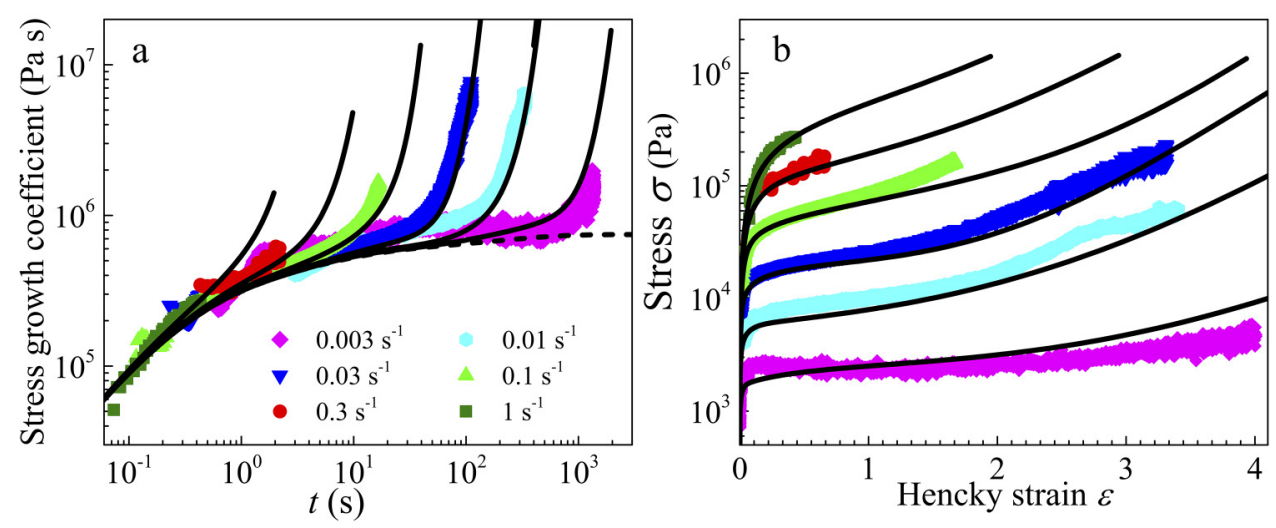

FIG. 11. (a) The time-dependent stress growth coefficient for varying extension rates, as measured using a filament stretching rheometer for UPyPEHA6 at a temperature of $T=343 \mathrm{~K}$. The stress growth coefficient corresponding to the linear viscoelastic envelope (LVE) is shown as a dashed line. The solid lines correspond to a description where each mode from a multi-mode Maxwell fit to the LVE data is assigned to an upper-convected Maxwell (UCM) model, as described in detail in the text. (b) The stress as a function of Hencky strain for the same measurements shown in panel a together with the fits to the UCM model. Note that data for the same extension rates are shown using the same symbols in panels a and $b$.

where $\kappa$ is the velocity gradient tensor and $I$ is the isotropic tensor. The dashed line in Fig. 11(a) represents the stress growth coefficient corresponding LVE which, in extension, is given by

$$
\eta_{\mathrm{E}}^{+}(t, \dot{\varepsilon})=3 \sum_{i} g_{i} \tau_{i}\left(1-\exp \left(-t / \tau_{i}\right)\right), \quad \dot{\varepsilon} \tau_{i} \ll 1 .
$$

It is clear that this simple model can provide an excellent description of the extension hardening up to the point of either sample fracture or termination of the experiment. This supports the suggestion, described above, that at these low flow rates the chain motion can be described using a Rouse-like model (given that the Rouse model is predicted to obey a multi-mode upper-convected Maxwell model in nonlinear flow [104]). Importantly, our results are fully consistent with the determination of the sticker dynamics based on modeling of our linear oscillatory shear rheology. Thus, it is useful to note that the nonlinear deformation can be described using the simple UCM model without recourse to more complicated modeling or simulations. Nevertheless, nonlinear rheology of supramolecular polymers in general is an area where considerable further work is needed in the future.

\section{CONCLUSIONS}

In this work, the linear viscoelastic response of a series of PEHA-based polymers with a systematically varied concentration of UPy-based supramolecular side chains was determined. The supramolecular interactions, i.e., hydrogen bonds between UPy groups, act as a second friction for polymer chains, and show a temperature dependence different from that of the segmental relaxation. We determined the range of validity of TTS for our samples and found that TTS works well for the pure nonsupramolecular polymer PEHA0 as well as the polymer with the lowest concentration of supramolecular UPy-based side chains. However, polymers with a higher concentration of UPy-based side chains are "thermorheologically complex" and TTS will work only over a limited time or frequency range. Our results stress the need for caution when constructing master curves in which data are shifted by many decades in frequency or time, since cumulative errors may lead to the wrong shape of the master curve; a careful comparison between TTS results and direct stress relaxation (or creep) data, that can extend the dynamic range, is thus generally recommended. We described the response of our supramolecular polymers using both a "classic" and a modified sticky-Rouse model. The modifications in the latter model were made to take into account (i) the random placement of stickers along the backbone, (ii) the contributions from dangling chain ends, and (iii) that the chain motion upon dissociation of a sticker and reassociation with a new coordination involves a finite sized "hop" of the chain. We found that both the "classic" sticky-Rouse model and our modified model describe data well in the low frequency range but the "classic" sticky-Rouse model is less successful in the intermediate plateau regime, where our modified model provides an improved description. It is, however, worth noting that the fits within the intermediate dynamic regime are still far from perfect. This indicates that additional physics, such as the distribution of mean sticker lifetimes, needs to be included for a further improved model. Finally, extensional nonlinear rheological measurements were performed on one of our supramolecular polymers. We observed that at short times before strain hardening occurs, the tensile (extensional) stress growth coefficient data followed the LVE envelope. However, for Hencky strain rates of $0.1 \mathrm{~s}^{-1}$ or below, strain hardening was observed at long times leading to tensile stress growth coefficients with magnitudes up to 10 times higher than those of the LVE envelope. We demonstrated that the upper-convected Maxwell model, based on a multi-mode Maxwell fit of the LVE data, described the nonlinear rheology data well.

\section{ACKNOWLEDGMENTS}

The work leading to these results has received funding from the People Programme (Marie Skłodowska-Curie Actions) of the European Union's Seventh Framework Programme (FP7/2007-2013) under REA grant agreement 
no. 607937-SUPOLEN project. Q.H. would like to acknowledge financial support from the Aage og Johanne Louis-Hansen Foundation. Data associated with this work are available from the Research Data Leeds repository under a CC-BY license at https://doi.org/10.5518/428.

\section{APPENDIX: COMPUTATION OF THE STRESS RELAXATION IN THE STOCHASTIC STICKY-ROUSE MODEL}

The contribution of the "fast" Rouse modes (on a timescale where the sticker configurations do not change) and the "slow" sticky modes (on a timescale where the dangling chain ends and strands of chains between sticker have relaxed) to the total stress relaxation, $G_{\text {stocha }}$, is written

$$
G_{\text {stocha }}(t)=G_{\text {fast }}(t)+G_{\text {sticky }}(t) .
$$

\section{1. "Fast" Rouse modes- $G_{\text {fast }}$}

Let us consider that the number of chains per unit volume is $n_{M} / N$, where $N$ is the degree of polymerization of the chain, and $n_{M}=\rho N_{\mathrm{A}} / M_{0}$ is the number of Rouse monomers per unit volume, with $\rho$ the polymer density, $N_{\mathrm{A}}$ the Avogadro constant, and $M_{0}$ the Rouse monomer molar mass. Thus, the "unit of modulus" per chain is

$$
\begin{aligned}
G_{\text {chain }}^{0} & =\frac{n_{M} k_{\mathrm{B}} T}{N} \\
& =\frac{\rho R T}{M},
\end{aligned}
$$

where we used the relation between the gas constant $R$ and the Boltzmann constant $k_{\mathrm{B}}, R=N_{\mathrm{A}} k_{\mathrm{B}}, T$ is the temperature, and $M=N M_{0}$ is the chain molar mass.

We have to consider the Rouse relaxation process of the segments "trapped" between two stickers, and that of the "dangling ends" (chain extremities). For each chain $k$, of molar mass $M$, we write the stress relaxation function of the "fast" Rouse modes as

$$
G_{\text {fast }, k}(t)=\frac{\rho R T}{M}\left(\tilde{G}_{\text {trapped }, k}(t)+\tilde{G}_{\text {ends }, k}(t)\right) .
$$

\section{a. Trapped chain segments $-\tilde{G}_{\text {trapped }}$}

For each chain $k$, the strand of chain of molar mass $M_{k, i}$ "trapped" between two stickers, $(i-1)$ and $i$, behaves as a Rouse chain with both ends fixed, and so relaxes via Rouse modes with relaxation time $\tau_{k, i}=N_{k, i}^{2} \tau_{0} / p^{2}$, with $p=\{1,2,3, \ldots\}$, and $N_{k, i} \equiv M_{k, i} / M_{0}$ the number of Rouse monomers in the $i$ th strand (of molar mass $M_{k, i}$ ) of the $k$ th chain. Hence,

$$
\tilde{G}_{\text {trapped }, k}(t)=\sum_{i=2}^{\mathrm{S}_{k}} \sum_{p=1}^{N_{k, i}} \exp \left(-\frac{t p^{2}}{N_{k, i}^{2} \tau_{0}}\right) .
$$

Note that the first sum excludes the chain ends. The secondsum cut-off, $N_{k, i}$, is chosen such that the fastest Rouse mode corresponds to relaxation time of a Rouse monomer, $\tau_{0}$.

\section{b. Chain ends $-\tilde{G}_{\text {ends }}(t)$}

For each chain $k$, the two end segments of molecular weight $M_{k, 1}$ and $M_{k,\left(\mathrm{~S}_{k}+1\right)}$, see Fig. 6 , behave as a Rouse chain with one end free and one end fixed (by the sticker). The Rouse model for such a chain section has boundary conditions $r=0$ at the fixed end, and $\partial r / \partial n=0$ at the free chain end. Consideration of the sinusoidal modes along the chain compatible with these boundary conditions reveals that these chain segments have a set of Rouse modes corresponding only to the "odd" modes of a free chain twice as long. Thus, the Rouse relaxation times are $\tau_{k, i}=\left(2 N_{k, i}\right)^{2} \tau_{0} / p^{2}$, with $p=\{1,3,5, \ldots\}$. The corresponding stress relaxation function is

$$
\tilde{G}_{\text {ends }, k}(t)=\sum_{i=\left\{1, S_{k}+1\right\}} \sum_{p=1, p_{\text {odd }}}^{N_{k, i}} \exp \left(-\frac{t p^{2}}{4 N_{k, i}^{2} \tau_{0}}\right) .
$$

\section{c. Storage and loss moduli- $G^{\prime}(\omega), G^{\prime \prime}(\omega)$}

For each chain $k$, the stress relaxation functions of the "trapped" and "end" strands are summarized as

$$
\begin{aligned}
\tilde{G}_{\text {fast }, k}^{\prime}(\omega)= & \sum_{i=2}^{\mathrm{S}_{k}} \sum_{p=1}^{N_{k, i}} \frac{\left(\omega N_{k, i}^{2} \tau_{0} p^{-2}\right)^{2}}{1+\left(\omega N_{k, i}^{2} \tau_{0} p^{-2}\right)^{2}} \\
& +\sum_{i=\left\{1, \mathrm{~S}_{k}+1\right\}} \sum_{p_{\text {odd }}}^{N_{k, i}} \frac{\left(4 \omega N_{k, i}^{2} \tau_{0} p^{-2}\right)^{2}}{1+\left(4 \omega N_{k, i}^{2} \tau_{0} p^{-2}\right)^{2}}, \\
\tilde{G}_{\text {fast }, k}^{\prime \prime}(\omega)= & \sum_{i=2}^{\mathrm{S}_{k}} \sum_{p=1}^{N_{k, i}} \frac{\omega N_{k, i}^{2} \tau_{0} p^{-2}}{1+\left(\omega N_{k, i}^{2} \tau_{0} p^{-2}\right)^{2}} \\
& +\sum_{i=\left\{1, \mathrm{~S}_{k}+1\right\}} \sum_{p_{\text {odd }}}^{N_{k, i}} \frac{4 \omega N_{k, i}^{2} \tau_{0} p^{-2}}{1+\left(4 \omega N_{k, i}^{2} \tau_{0} p^{-2}\right)^{2}} .
\end{aligned}
$$

The expression of the total elastic and loss moduli is obtained by summing the"trapped" and "end" contributions of the "fast Rouse" motion, and summing over the $\mathrm{C}$ chains of identical molecular weight, but of different number of stickers placed randomly along the chain backbone

$$
\begin{aligned}
& G_{\text {fast }}^{\prime}(\omega)=\frac{\rho R T}{M} \frac{1}{\mathrm{C}} \sum_{k=1}^{\mathrm{C}} \tilde{G}_{\text {fast }, k}^{\prime}(\omega), \\
& G_{\text {fast }}^{\prime \prime}(\omega)=\frac{\rho R T}{M} \frac{1}{\mathrm{C}} \sum_{k=1}^{\mathrm{C}} \tilde{G}_{\text {fast }, k}^{\prime \prime}(\omega) .
\end{aligned}
$$

\section{d. Polydispersity}

The above Eqs. (A8) and (A9) assume a perfectly monodisperse system, i.e., all chains have the same molar mass $M$. We can generalize to the polydisperse case. If we assume that the molecular mass distribution is discretized into a set 
of $q$ modes, $\left\{\left(w_{\ell}, M_{\ell}\right)\right\}, \ell=\{1, \ldots, q\}$, and that $\mathrm{C}_{\ell}$ chains of molar mass $M_{\ell}$ are generated as described above, then the elastic and loss moduli are written

$$
\begin{aligned}
& G_{\text {fast }}^{\prime}(\omega)=\sum_{\ell=1}^{q} w_{\ell} \frac{\rho R T}{M_{\ell}} \frac{1}{\mathrm{C}_{\ell}} \sum_{k=1}^{\mathrm{C}_{\ell}} \tilde{G}_{\text {fast }, k, \ell}^{\prime}(\omega), \\
& G_{\text {fast }}^{\prime \prime}(\omega)=\sum_{\ell=1}^{q} w_{\ell} \frac{\rho R T}{M_{\ell}} \frac{1}{\mathrm{C}_{\ell}} \sum_{k=1}^{\mathrm{C}_{\ell}} \tilde{G}_{\text {fast }, k, \ell}^{\prime \prime}(\omega) .
\end{aligned}
$$

Note that for each molecular mass $M_{\ell}$ we need to generate an ensemble of $\mathrm{C}_{\ell}$ chains, as described in Sec. IV B.

\section{2. "Sticky" modes- $G_{\text {sticky }}(t)$}

We now describe a stochastic algorithm which we use to model the motion of chains on long time scales, and which we can use to obtain the relaxation spectrum for the slow chain modes.

\section{a. Initial spatial configuration}

Each chain of the simulation is initialized to have a Gaussian configuration. We start by positioning the first sticker at an arbitrary position, $R_{0 \text {,init }}$, (e.g.,$R_{0, \text { init }}=0$ ) and define the position of the following sticker, $i$, (relative to the previous sticker) by subsequently generating a random vector, $\Delta R_{i, \text { init }}$, sampled from the Gaussian probability distribution

$$
p\left(\Delta R_{i, \text { init }}\right)=\left(\frac{3}{2 \pi b^{2} N_{i}}\right)^{3 / 2} \exp \left(-\frac{3\left(\Delta R_{i, \text { init }}\right)^{2}}{2 b^{2} N_{i}}\right),
$$

where $N_{i} \equiv M_{i} / M_{0}$ is the number of Rouse monomers on the strand connecting the stickers $(i-1)$ and $i$, and $M_{0}$ the molar mass of a Rouse monomer. Then, we place the sticker $i$ at the initial position $R_{i, \text { init }}$ such that

$$
R_{i, \text { init }}=R_{i-1, \text { init }}+\Delta R_{i, \text { init }}
$$

\section{b. Sticker "hop"}

We made the assumption that the average time during which the stickers stay attached is much longer than any of the internal Rouse relaxation times of the segments of chain delimited by the stickers. We consider that once a sticker, $i$, detaches, it takes a "hop" to a new position which is a vector $\Delta R$ from a mean position $\bar{R}_{i}$, where it reattaches, see Fig. 8 . As described in Sec. IV A, this "hop" motion is the result of the change of partner that a sticker undergoes, on average, every $\tau_{\mathrm{s}}$. Here we assume that between detachment and reattachment, the sticker is able to explore the full configurational space available to it, given that it is constrained by the chain and its neighboring stickers do not move, see Fig. 8. The mean position $\bar{R}_{i}$, around which the sticker reattaches, is defined by the molecular weight of the strands $\left(M_{i}, M_{i+1}\right)$ that are connected to the sticker and by the position of the neighboring stickers $\left(R_{i-1}, R_{i+1}\right)$ as the weighted average position,

$$
\bar{R}_{i}=\frac{M_{i+1} R_{i-1}+M_{i} R_{i+1}}{M_{i}+M_{i+1}} .
$$

Additionally, we obtain the probability distribution function of the "hop size," $\Delta R_{i}$, (i.e., how much far from the average position, $\bar{R}_{i}$, the sticker will attach) as

$$
p\left(\Delta R_{i}\right)=\left(\frac{1}{2 \pi \sigma_{i}^{2}}\right)^{3 / 2} \exp \left(-\frac{\left(\Delta R_{i}\right)^{2}}{2 \sigma_{i}^{2}}\right),
$$

where the variance is $\sigma_{i}^{2}=k_{\mathrm{B}} T / k_{\mathrm{eff}, i}$, with $k_{\mathrm{eff}, i}$ the effective spring constant associated with the sticker $i$, which depends on the neighboring chain segments

$$
k_{\mathrm{eff}, i}=\frac{3 k_{\mathrm{B}} T}{b^{2} N_{i}}+\frac{3 k_{\mathrm{B}} T}{b^{2} N_{i+1}},
$$

where $b$ is the statistical length of a Rouse monomer and $N_{i}=M_{i} / M_{0}$ is the number of Rouse monomers in $M_{i}$.

Assuming isotropy of the "hop," each coordinate $\left(\Delta x_{i}, \Delta y_{i}, \Delta z_{i}\right)$ of $\Delta R_{i}$ follows the same probability distribution

$$
p\left(\Delta x_{i}\right)=\left(\frac{1}{2 \pi \sigma_{i}^{2}}\right)^{1 / 2} \exp \left(-\frac{\left(\Delta x_{i}\right)^{2}}{2 \sigma_{i}^{2}}\right) .
$$

Therefore, when a sticker detaches and reattaches, its new position, $R_{i}^{\text {new }}$ is given by

$$
R_{i}^{\text {new }}=\bar{R}_{i}+\Delta R_{i}
$$

For the first sticker $(i=1)$ and last sticker $(i=\mathrm{S})$, we use

$$
\bar{R}_{1}=R_{2} \quad \text { and } \quad \bar{R}_{\mathrm{S}}=R_{\mathrm{S}-1},
$$

and for the effective spring constants Eq. (A16), we use

$$
k_{\mathrm{eff}, 1}=\frac{3 k_{\mathrm{B}} T}{b^{2} N_{2}} \quad \text { and } \quad k_{\mathrm{eff}, \mathrm{S}}=\frac{3 k_{\mathrm{B}} T}{b^{2} N_{\mathrm{S}}} .
$$

The above rules ensure that the chains continue to obey the correct equilibrium Gaussian chain distribution upon hopping. We assume the time between detachment and reattachment is negligible.

\section{c. Sticker detachment dynamics}

For each molecular weight component $\ell$, we have generated $\mathrm{C}_{\ell}$ chains (of molar mass $M_{\ell}$ ) with a certain amount of stickers placed randomly along the chain, according to Eq. (3). The total number of stickers over the $C_{\ell}$ chains is $\mathrm{S}_{\ell, \text { tot }}=\sum_{k=1}^{\mathrm{C}_{\ell}} \mathrm{S}_{\ell, k}$.

For a given sticker, the cumulative distribution function for the detachment time $t_{\mathrm{d}}$ of that sticker (time after which an associated sticker detaches) is

$$
p\left(t_{\mathrm{d}} \leq t\right)=1-\exp \left(-\frac{t}{\tau_{\mathrm{s}}}\right)
$$

Therefore, the probability that a sticker did not detach after a 
time $t$ is $p\left(t \leq t_{\mathrm{d}}\right)=\exp \left(-t / \tau_{\mathrm{s}}\right)$. Hence, the probability that none of the $\mathrm{S}_{\ell, \text { tot }}$ stickers have detached after a time $t$ is

$$
\left[p\left(t \leq t_{\mathrm{d}}\right)\right]^{\mathrm{S}_{\ell, \mathrm{tot}}}=\exp \left(-\frac{\mathrm{S}_{\ell, \text { tot }} t}{\tau_{\mathrm{s}}}\right) .
$$

We conclude that the probability density function of the detachment time of the first sticker to detach among the $S_{\ell, \text { tot }}$ stickers is

$$
p\left(t_{\mathrm{d}, \text { first }}\right)=\frac{\mathbf{S}_{\ell, \text { tot }}}{\tau_{\mathrm{s}}} \exp \left(-\frac{\mathbf{S}_{\ell, \text { tot }} t_{\text {d,first }}}{\tau_{\mathrm{s}}}\right) .
$$

Given a uniformly distributed (pseudo) random number $0<\theta<1$, we generate from Eq. (A23) a time, $t_{\mathrm{d} \text {,first }}$, after which a first sticker detaches:

$$
t_{\mathrm{d}, \text { first }}=-\tau_{\mathrm{s}} \ln (\theta) / \mathrm{S}_{\ell, \text { tot }} .
$$

Then, we choose a sticker randomly among the $S_{\ell, \text { tot }}$ stickers and allow it to make a "hop" as described in Eq. (A18). We then repeat this process many times to find the next detachment time among the stickers, selecting a random sticker to move each time. The simulation time advances by steps of (random) size $t_{\mathrm{d} \text {,first }}$, which is on average decreasing with increasing $S_{\ell \text { tot }}$, i.e., the simulation slows down as the chain molecular mass $M_{\ell}$ increases. Therefore, it is sometimes necessary to reduce $\mathrm{C}_{\ell}$ for the highest molecular mass.

\section{d. Stress tensor and stress relaxation}

A microscopic expression for the stress tensor is [104]

$$
\sigma_{\alpha \beta}=\frac{1}{V} \sum_{\text {springs }, m} F_{m \alpha} R_{m \beta},
$$

where the summation is made over all the springs in the system, the Greek letters are the Cartesian coordinates, $F_{m}=3 k_{\mathrm{B}} T R_{m} / N_{m} b^{2}$ is the entropic spring force acting in the $m$ th strand, $R_{m}$ is the vector connecting the two beads neighboring the strand $m$, and $V=\mathrm{CN} / n_{M}$ is the volume occupied by the $\mathrm{C}$ chains.

For each mode $\ell$ of the molecular weight distribution, Eq. (A25) can be written in terms of a sum over the $\mathrm{C}_{\ell}$ chains and, for each chain $k$, a sum over the $\mathrm{S}_{\ell, k}-1$ chain segments trapped between two stickers

$$
\begin{aligned}
\sigma_{\alpha \beta, \ell} & =\frac{1}{V} \sum_{k=1}^{\mathrm{C}_{\ell}} \sum_{i=2}^{\mathrm{S}_{\ell, k}} \frac{3 k_{\mathrm{B}} T}{N_{i} b^{2}} R_{\ell, i, \alpha} R_{\ell, i, \beta} \\
& =\frac{\rho R T}{M_{\ell}} 1 \mathrm{C}_{\ell} \sum_{k=1}^{\mathrm{C}_{\ell}} \sum_{i=2}^{\mathrm{S}_{\ell, k}} \frac{3}{N_{i} b^{2}} R_{\ell, i, \alpha} R_{\ell, i, \beta},
\end{aligned}
$$

where $M_{\ell}$ is the $\ell$ th molar mass mode of the molecular weight distribution. Note that the two end segments (polymers ends) are excluded from this stress expression as we consider that they are relaxed and their contributions were already accounted for in $G_{\text {fast }}$, see section 1 of this appendix.

In computer simulations, the most convenient way of evaluating the stress relaxation is by using the fluctuation- dissipation theorem $[102,103]$. For each mode $\ell$, we have:

$$
\begin{aligned}
G_{\text {sticky }, \ell}(t) & =\frac{V}{k_{\mathrm{B}} T} \frac{1}{t_{\mathrm{sim}}-t} \int_{0}^{t_{\mathrm{sim}}-t} \sigma_{x y, \ell}(t+\tau) \sigma_{x y, \ell}(\tau) \mathrm{d} \tau \\
& =\frac{V}{k_{\mathrm{B}} T}\left\langle\sigma_{x y, \ell}(t+\tau) \sigma_{x y, \ell}(\tau)\right\rangle \\
& =\mathrm{C}_{\ell} \frac{M_{\ell}}{\rho R T}\left\langle\sigma_{x y, \ell}(t+\tau) \sigma_{x y, \ell}(\tau)\right\rangle,
\end{aligned}
$$

where $x y$ is any two orthogonal directions, and $t_{\text {sim }}$ the total simulation time.

Since our system is isotropic, one can average over different directions defining the pair of perpendicular axis $(x y)$. In isotropic systems there are two arbitrary angles to select the direction of $x$ axis and one more angle to select the direction of $y$-axis perpendicular to it. Averaging over these three angles gives the following result $[102,103]$

$$
\begin{aligned}
G_{\text {sticky }, \ell}(t)= & \frac{M_{\ell} \mathrm{C}_{\ell}}{5 \rho R T}\left(\left\langle\sigma_{x y, \ell}(t) \sigma_{x y, \ell}(\tau)\right\rangle+\left\langle\sigma_{y z, \ell}(t) \sigma_{y z, \ell}(\tau)\right\rangle\right. \\
& \left.+\left\langle\sigma_{z x, \ell}(t) \sigma_{z x, \ell}(\tau)\right\rangle\right) \\
& +\frac{M_{\ell} \mathrm{C}_{\ell}}{30 \rho R T}\left(\left\langle N_{x y, \ell}(t) N_{x y, \ell}(\tau)\right\rangle+\left\langle N_{x z, \ell}(t) N_{x z, \ell}(\tau)\right\rangle\right. \\
& \left.+\left\langle N_{y z, \ell}(t) N_{y z, \ell}(\tau)\right\rangle\right),
\end{aligned}
$$

where $N_{\alpha \beta, \ell}=\sigma_{\alpha \alpha, \ell}-\sigma_{\beta \beta, \ell}$. Using the latter expression instead of Eq. (A27) improves the statistical accuracy of the results.

In order to evaluate correlation functions in simulations, we use a multiple-tau correlator algorithm proposed by Ramirez et al. [103].

\section{e. Polydispersity}

If we assume that the molecular mass distribution is discretized into a set of $q$ modes, $\left\{\left(w_{\ell}, M_{\ell}\right)\right\}, \ell=\{1,, q\}$, then we compute $G_{\text {sticky }}$ as

$$
G_{\text {sticky }}=\sum_{\ell=1}^{q} w_{\ell} G_{\text {sticky }, \ell},
$$

where $G_{\text {sticky, } \ell}$ is computed using Eq. (A28). Note that for each molecular mass $M_{\ell}$, we need to generate an ensemble of $\mathrm{C}_{\ell}$ chains, as described in Sec. IV B.

\section{References}

[1] Cui, J., D. Wang, K. Koynov, and A. del Campo, "2-Ureido-4-pyrimidone-based hydrogels with multiple responses," ChemPhysChem 14, 2932-2938 (2013).

[2] Appel, W. P. J., G. Portale, E. Wisse, P. Y. W. Dankers, and E. W. Meijer, "Aggregation of ureido-pyrimidinone supramolecular thermoplastic elastomers into nanofibers: A kinetic analysis," Macromolecules 44, 6776-6784 (2011).

[3] Yamauchi, K., J. R. Lizotte, D. M. Hercules, M. J. Vergne, and T. E. Long, "Combinations of microphase separation and terminal multiple hydrogen bonding in novel macromolecules," J. Am. Chem. Soc. 124, 8599-8604 (2002). 
[4] Kautz, H., D. J. M. van Beek, R. P. Sijbesma, and E. W. Meijer, "Cooperative end-to-end and lateral hydrogen-bonding motifs in supramolecular thermoplastic elastomers," Macromolecules 39, 4265-4267 (2006).

[5] Pellizzaro, M. L., S. A. Barrett, J. Fisher, and A. J. Wilson, "Design, synthesis and binding studies of a novel quadruple ADDA hydrogenbond array," Org. Biomol. Chem. 10, 4899-4906 (2012).

[6] Lou, N., Y. Wang, X. Li, H. Li, P. Wang, C. Wesdemiotis, A. P. Sokolov, and H. Xiong, "Dielectric relaxation and rheological behavior of supramolecular polymeric liquid," Macromolecules 46, 3160-3166 (2013).

[7] Chen, S., and W. H. Binder, "Dynamic ordering and phase segregation in hydrogen-bonded polymers," Acc. Chem. Res. 49, 1409-1420 (2016).

[8] Hirschberg, J. K., L. Brunsveld, A. Ramzi, J. A. Vekemans, R. P. Sijbesma, and E. Meijer, "Helical self-assembled polymers from cooperative stacking of hydrogen-bonded pairs," Nature 407, 167-170 (2000).

[9] Brunsveld, L., J. Vekemans, J. Hirschberg, R. Sijbesma, and E. Meijer, "Hierarchical formation of helical supramolecular polymers via stacking of hydrogen-bonded pairs in water," Proc. Natl. Acad. Sci. 99, 4977-4982 (2002).

[10] Zhu, B., N. Jasinski, A. Benitez, M. Noack, D. Park, A. S. Goldmann, C. Barner-Kowollik, and A. Walther, "Hierarchical nacre mimetics with synergistic mechanical properties by control of molecular interactions in self-healing polymers," Angew. Chem. Int. Ed. 54, 8653-8657 (2015).

[11] Chirila, T. V., H. H. Lee, M. Oddon, M. M. Nieuwenhuizen, I. Blakey, and T. M. Nicholson, "Hydrogen-bonded supramolecular polymers as self-healing hydrogels: Effect of a bulky adamantyl substituent in the ureido-pyrimidinone monomer," J. Appl. Polym. Sci. 131, 39932 (2014).

[12] Mather, B. D., C. L. Elkins, F. L. Beyer, and T. E. Long, "Morphological analysis of telechelic ureidopyrimidone functional hydrogen bonding linear and star-shaped poly(ethylene-co-propylene)s," Macromol. Rapid Commun. 28, 1601-1606 (2007).

[13] Wilson, A. J., "Non-covalent polymer assembly using arrays of hydrogen-bonds," Soft Matter 3, 409-425 (2007).

[14] Feldman, K. E., M. J. Kade, T. F. A. de Greef, E. W. Meijer, E. J. Kramer, and C. J. Hawker, "Polymers with multiple hydrogen-bonded end groups and their blends," Macromolecules 41, 4694-4700 (2008).

[15] Gooch, A., N. S. Murphy, N. H. Thomson, and A. J. Wilson, "Side-chain supramolecular polymers employing conformer independent triple hydrogen bonding arrays," Macromolecules 46, 9634-9641 (2013).

[16] Beijer, F. H., R. P. Sijbesma, H. Kooijman, A. L. Spek, and E. W. Meijer, "Strong dimerization of ureidopyrimidones via quadruple hydrogen bonding," J. Am. Chem. Soc. 120, 6761-6769 (1998).

[17] Brzezski, M., and T. Biela, "Supramolecular polylactides by the cooperative interaction of the end groups and stereocomplexation," Macromolecules 48, 2994-3004 (2015).

[18] Folmer, B. J. B., R. P. Sijbesma, R. M. Versteegen, J. A. J. van der Rijt, and E. W. Meijer, "Supramolecular polymer materials: Chain extension of telechelic polymers using a reactive hydrogen-bonding synthon," Adv. Mater. 12, 874-878 (2000).

[19] Heinzmann, C., I. Lamparth, K. Rist, N. Moszner, G. L. Fiore, and C. Weder, "Supramolecular polymer networks made by solvent-free copolymerization of a liquid 2-ureido-4[1H]-pyrimidinone methacrylamide," Macromolecules 48, 8128-8136 (2015).
[20] Elkins, C. L., T. Park, M. G. McKee, and T. E. Long, "Synthesis and characterization of poly(2-ethylhexyl methacrylate) copolymers containing pendant, self-complementary multiple-hydrogen-bonding sites,” J. Polym. Sci. Part A: Polym. Chem. 43, 4618-4631 (2005).

[21] Bobade, S., Y. Wang, J. Mays, and D. Baskaran, "Synthesis and characterization of ureidopyrimidone telechelics by CuAAC "Click" reaction: Effect of $\mathrm{Tg}$ and polarity," Macromolecules 47, 5040-5050 (2014).

[22] Chino, K., and M. Ashiura, "Themoreversible cross-linking rubber using supramolecular hydrogen-bonding networks," Macromolecules 34, 9201-9204 (2001).

[23] Guo, M., L. M. Pitet, H. M. Wyss, M. Vos, P. Y. W. Dankers, and E. W. Meijer, "Tough stimuli-responsive supramolecular hydrogels with hydrogen-bonding network junctions," J. Am. Chem. Soc. 136, 6969-6977 (2014).

[24] Ware, T., K. Hearon, A. Lonnecker, K. L. Wooley, D. J. Maitland, and W. Voit, "Triple-shape memory polymers based on self-complementary hydrogen bonding," Macromolecules 45, 1062-1069 (2012).

[25] Gooch, A., C. Nedolisa, K. A. Houton, C. I. Lindsay, A. Saiani, and A. J. Wilson, "Tunable self-assembled elastomers using triply hydrogen-bonded arrays," Macromolecules 45, 4723-4729 (2012).

[26] Hofmeier, H., and U. S. Schubert, "Recent developments in the supramolecular chemistry of terpyridine-metal complexes," Chem. Soc. Rev. 33, 373-99 (2004).

[27] Andres, P. R., H. Hofmeier, and U. S. Schubert, "Complexation parameters of terpyridine-metal complexes," in Metal-Containing and Metallosupramolecular Polymers and Materials, edited by U. S. Schubert, G. R. Newkome, and I. Manners (ACS, Washington, DC, 2006), Chap. 11, pp. 141-156.

[28] Hoogenboom, R., J. Huskens, and U. S. Schubert, "Grid forming metal coordinating macroligands: Synthesis and complexation," in Metal-Containing and Metallosupramolecular Polymers and Materials, edited by U. S. Schubert, G. R. Newkome, and I. Manners (ACS, Washington, DC, 2006), Vol. 928, pp. 63-71.

[29] Rowan, S. J., and J. B. Beck, "Metal-ligand induced supramolecular polymerization: A route to responsive materials," Faraday Discuss. 128, 43-53 (2005).

[30] Shunmugam, R., G. J. Gabriel, K. A. Aamer, and G. N. Tew, "Metal-ligand-containing polymers: Terpyridine as the supramolecular unit," Macromol. Rapid Commun. 31, 784-93 (2010).

[31] Han, F. S., M. Higuchi, and D. G. Kurth, "Metallo-supramolecular polymers based on functionalized bis-terpyridines as novel electrochromic materials," Adv. Mater. 19, 3928-3931 (2007).

[32] Beck, J. B., and S. J. Rowan, "The preparation of metallosupramolecular polymers and gels by utilizing 2,6- bis -(1-methyl-benzimidazolyl)pyridine metal ion interactions," in Metal-Containing and Metallosupramolecular Polymers and Materials (ACS, Washington, DC, 2006), pp. 97-112.

[33] Hofmeier, H., M. Wouters, D. Wouters, and U. S. Schubert, "Thermal stability, rheology, and morphology of metallosupramolecular polymers based on bis-terpyridine-ruthenium(II) complexes," Metal-Containing and Metallosupramolecular Polymers and Materials (ACS, Washington, DC, 2006), pp. 113-125.

[34] Kumpfer, J. R., J. J. Wie, J. P. Swanson, F. L. Beyer, M. E. Mackay, and S. J. Rowan, "Influence of metal ion and polymer core on the melt rheology of metallosupramolecular films," Macromolecules $\mathbf{4 5}$, 473-480 (2012).

[35] Hofmeier, H., R. Hoogenboom, M. E. L. Wouters, and U. S. Schubert, "High molecular weight supramolecular polymers containing both terpyridine metal complexes and ureidopyrimidinone 
quadruple hydrogen-bonding units in the main chain," J. Am. Chem. Soc. 127, 2913-2921 (2005).

[36] Schubert, U. S., O. Hien, and C. Eschbaumer, "Functionalized polymers with metal complexing segments: A simple and high-yield entry towards 2,2' : 6', 2" -terpyridine-based oligomers," Macromol. Rapid Commun. 21, 1156-1161 (2000).

[37] Schmatloch, S., and U. S. Schubert, "Engineering with metallosupramolecular polymers: Linear coordination polymers and networks," Macromol. Symp. 199, 483-498 (2003).

[38] Goldansaz, H., Q. Voleppe, S. Pioge, C. A. Fustin, J. F. Gohy, J. Brassinne, D. Auhl, and E. van Ruymbeke, "Controlling the melt rheology of linear entangled metallo-supramolecular polymers," Soft Matter 11, 762-74 (2015).

[39] Schwartz, G. A., R. Bergman, J. Mattsson, and J. Swenson, "Dielectric relaxation studies of poly(propylene glycol) confined in vermiculite clay,” Eur. Phys. J. E 12, S113-S116 (2003).

[40] Gonzlez, L., A. Ladegaard Skov, and S. Hvilsted, "Ionic networks derived from the protonation of dendritic amines with carboxylic acid end-functionalized PEGs,” J. Polym. Sci. Part A: Polym. Chem. 51, 1359-1371 (2013).

[41] Chen, Q., G. J. Tudryn, and R. H. Colby, "Ionomer dynamics and the sticky Rouse model,” J. Rheol. 57, 1441-1462 (2013).

[42] Ling, G. H., Y. Wang, and R. A. Weiss, "Linear viscoelastic and uniaxial extensional rheology of alkali metal neutralized sulfonated oligostyrene ionomer melts," Macromolecules 45, 481-490 (2012).

[43] Stadler, F. J., W. Pyckhout-Hintzen, J.-M. Schumers, C.-A. Fustin, J.-F. Gohy, and C. Bailly, "Linear viscoelastic rheology of moderately entangled telechelic polybutadiene temporary networks," Macromolecules 42, 6181-6192 (2009).

[44] Chen, Q., H. Masser, H.-S. Shiau, S. Liang, J. Runt, P. C. Painter, and R. H. Colby, "Linear viscoelasticity and fourier transform infrared spectroscopy of polyether-ester-sulfonate copolymer ionomers," Macromolecules 47, 3635-3644 (2014).

[45] Roland, C. M., K. L. Ngai, P. G. Santangelo, X. H. Qiu, M. D. Ediger, and D. J. Plazek, "Temperature dependence of segmental and terminal relaxation in atactic polypropylene melts," Macromolecules 34, 6159-6160 (2001).

[46] Bosman, A. W., R. P. Sijbesma, and E. W. Meijer, "Supramolecular polymers at work," Mater. Today 7, 34-39 (2004).

[47] Sijbesma, R. P., F. H. Beijer, L. Brunsveld, B. J. B. Folmer, J. H. K. K. Hirschberg, R. F. M. Lange, J. K. L. Lowe, and E. W. Meijer, "Reversible polymers formed from self-complementary monomers using quadruple hydrogen bonding," Science 278, 1601-1604 (1997).

[48] de Greef, T. F. A., and E. W. Meijer, "Materials science: Supramolecular polymers," Nature 453, 171-173 (2008).

[49] Wei, M., M. Zhan, D. Yu, H. Xie, M. He, K. Yang, and Y. Wang, "Novel poly(tetramethylene ether)glycol and poly( $\varepsilon$-caprolactone) based dynamic network via quadruple hydrogen bonding with tripleshape effect and self-healing capacity," ACS. Appl. Mater. Interfaces 7, 2585-2596 (2015).

[50] Cui, J., and A. d. Campo, "Multivalent H-bonds for self-healing hydrogels," Chem. Commun. 48, 9302-9304 (2012).

[51] Herbst, F., D. Dhler, P. Michael, and W. H. Binder, "Self-healing polymers via supramolecular forces," Macromol. Rapid Commun. 34, 203-220 (2013).

[52] Herbst, F., S. Seiffert, and W. H. Binder, "Dynamic supramolecular poly(isobutylene)s for self-healing materials," Polym. Chem. 3, 3084 (2012).

[53] Sivakova, S., D. A. Bohnsack, M. E. Mackay, P. Suwanmala, and S. J. Rowan, "Utilization of a combination of weak hydrogen-bonding interactions and phase segregation to yield highly thermosensitive supramolecular polymers," J. Am. Chem. Soc. 127, 18202-18211 (2005).

[54] Li, S., F. Huo, Q. Li, C. Gao, Y. Su, and W. Zhang, "Synthesis of a doubly thermo-responsive schizophrenic diblock copolymer based on poly[N-(4-vinylbenzyl)-N,N-diethylamine] and its temperaturesensitive flip-flop micellization," Polym. Chem. 5, 3910-3918 (2014).

[55] Jaeger, C. W., and C. R. King, Phase change ink composition, US Patent No. 6,245,135 (2001).

[56] Shimada, M., T. Kawanishi, K. Murakami, T. Aruga, H. Uemura, and K. Nagai, Aqueous ink composition, US Patent No. 4,711,668 (1987).

[57] Pappas, P. S., A. Monk, S. Saraiya, and J. Huang, Imageable element and composition comprising thermally reversible polymers, US Patent No. 6,911,296 (2005).

[58] Asawa, Y., Y. Ishizuka, E. Hayakawa, and S. P. Pappas, Two-layer imageable element comprising thermally reversible polymers, US Patent No. 6,902,860 (2005).

[59] Mougin, N., A. Livoreil, and J. Mondet, Cosmetic composition forming after application a supramolecular polymer, US Patent No. 8,354,097 (2013).

[60] Frechet, J. M., D. Hajduk, E. Khoshdel, M. Liu, R. B. Nielsen, E. S. Reid, and K. L. Rutherford, Cosmetic and personal care compositions, US Patent 6,663,855 (2003).

[61] Eling, B., and C. Lindsay, Supramolecular polymer forming polymer, US Patent App. No. 10/444,612 (2003).

[62] Jansen, J., J. Loontjes, and B. Plum, Supramolecular compound, EP Patent App. No. EP20,000,200,583 (2000).

[63] Lewis, C. L., and M. Anthamatten, "Synthesis, swelling behavior, and viscoelastic properties of functional poly(hydroxyethyl methacrylate) with ureidopyrimidinone side-groups," Soft Matter 9, 4058-4066 (2013).

[64] Lewis, C. L., K. Stewart, and M. Anthamatten, "The influence of hydrogen bonding side-groups on viscoelastic behavior of linear and network polymers," Macromolecules 47, 729-740 (2014).

[65] Shabbir, A., I. Javakhishvili, S. Cerveny, S. Hvilsted, A. L. Skov, O. Hassager, and N. J. Alvarez, "Linear viscoelastic and dielectric relaxation response of unentangled UPy-based supramolecular networks," Macromolecules 49, 3899-3910 (2016).

[66] Feldman, K. E., M. J. Kade, E. W. Meijer, C. J. Hawker, and E. J. Kramer, "Model transient networks from strongly hydrogen-bonded polymers," Macromolecules 42, 9072-9081 (2009).

[67] Gold, B., C. Hvelmann, N. Luhmann, W. Pyckhout-Hintzen, A. Wischnewski, and D. Richter, "The microscopic origin of the rheology in supramolecular entangled polymer networks," J. Rheol. 61, 1211-1226 (2017).

[68] Ferry, J. D., Viscoelastic Properties of Polymers (John Wiley \& Sons, 1980).

[69] Shinozuka, M., "Thermorheologically simple viscoelastic materials," AIAA J. 3, 375-377 (1965).

[70] Stadler, F. J., W. Pyckhout-Hintzen, J.-M. Schumers, C.-A. Fustin, J.-F. Gohy, and C. Bailly, "Linear viscoelastic rheology of moderately entangled telechelic polybutadiene temporary networks," Macromolecules 42, 6181-6192 (2009).

[71] Plazek, D. J., "Temperature dependence of the viscoelastic behavior of polystyrene," J. Phys. Chem. 69, 3480-3487 (1965).

[72] Plazek, D. J., "The temperature dependence of the viscoelastic behavior of poly(vinyl acetate)," Polym. J. 12, 43-53 (1980).

[73] Ding, Y., and A. P. Sokolov, "Breakdown of time-temperature superposition principle and universality of chain dynamics in polymers," Macromolecules 39, 3322-3326 (2006).

[74] Plazek, D. J., I. C. Chay, K. L. Ngai, and C. M. Roland, "Viscoelastic properties of polymers. 4. Thermorheological 
complexity of the softening dispersion in polyisobutylene," Macromolecules 28, 6432-6436 (1995).

[75] Seiffert, S., "Effect of supramolecular interchain sticking on the lowfrequency relaxation of transient polymer networks," Macromol. Rapid Commun. 37, 257-264 (2016).

[76] Shivokhin, M. E., T. Narita, L. Talini, A. Habicht, S. Seiffert, T. Indei, and J. D. Schieber, "Interplay of entanglement and association effects on the dynamics of semidilute solutions of multisticker polymer chains," J. Rheol. 61, 1231-1241 (2017).

[77] UneyamaT., S. Suzuki, and H. Watanabe, "Concentration dependence of rheological properties of telechelic associative polymer solutions," Phys. Rev. E 86, 031802 (2012).

[78] Annable, T., R. Buscall, R. Ettelaie, and D. Whittlestone, "The rheology of solutions of associating polymers: Comparison of experimental behavior with transient network theory," J. Rheol. 37, 695-726 (1993).

[79] Green, M. S., and A. V. Tobolsky, "A new approach to the theory of relaxing polymeric media,” J. Chem. Phys. 14, 80-92 (1946).

[80] Leibler, L., M. Rubinstein, and R. H. Colby, "Dynamics of reversible networks," Macromolecules 24, 4701-4707 (1991).

[81] Baxandall, L. G., "Dynamics of reversibly crosslinked chains," Macromolecules 22, 1982-1988 (1989).

[82] Rubinstein, M., and A. N. Semenov, "Thermoreversible gelation in solutions of associating polymers. 2. Linear dynamics," Macromolecules 31, 1386-1397 (1998).

[83] Ahmadi, M., L. G. Hawke, H. Goldansaz, and E. van Ruymbeke, "Dynamics of entangled linear supramolecular chains with sticky side groups: Influence of hindered fluctuations," Macromolecules 48, 7300-7310 (2015).

[84] Rubinstein, M., and A. N. Semenov, "Dynamics of entangled solutions of associating polymers," Macromolecules 34, 1058-1068 (2001).

[85] van Ruymbeke, E., K. Orfanou, M. Kapnistos, H. Iatrou, M. Pitsikalis, N. Hadjichristidis, D. Lohse, and D. Vlassopoulos, "Entangled dendritic polymers and beyond: Rheology of symmetric Cayley-tree polymers and macromolecular self-assemblies," Macromolecules 40, 5941-5952 (2007).

[86] van Ruymbeke, E., D. Vlassopoulos, M. Mierzwa, T. Pakula, D. Charalabidis, M. Pitsikalis, and N. Hadjichristidis, "Rheology and structure of entangled telechelic linear and star polyisoprene melts," Macromolecules 43, 4401-4411 (2010).

[87] Boudara, V. A., and D. J. Read, "Stochastic and preaveraged nonlinear rheology models for entangled telechelic star polymers," J. Rheol. 61, 339-362 (2017).

[88] Tang, S., M. Wang, and B. D. Olsen, "Anomalous self-diffusion and sticky Rouse dynamics in associative protein hydrogels," J. Am. Chem. Soc. 137, 3946-3957 (2015).

[89] Stadler, F. J., T. Still, G. Fytas, and C. Bailly, "Elongational rheology and Brillouin light scattering of entangled telechelic polybutadiene based temporary networks," Macromolecules 43, 7771-7778 (2010).
[90] Ling, G. H., Y. Wang, and R. Weiss, "Linear viscoelastic and uniaxial extensional rheology of alkali metal neutralized sulfonated oligostyrene ionomer melts," Macromolecules 45, 481-490 (2011).

[91] Shabbir, A., H. Goldansaz, O. Hassager, E. van Ruymbeke, and N. J. Alvarez, "Effect of hydrogen bonding on linear and nonlinear rheology of entangled polymer melts," Macromolecules 48, 5988-5996 (2015).

[92] Tudryn, G. J., M. V. O'Reilly, S. Dou, D. R. King, K. I. Winey, J. Runt, and R. H. Colby, "Molecular mobility and cation conduction in polyether-ester-sulfonate copolymer ionomers," Macromolecules $\mathbf{4 5}$, 3962-3973 (2012).

[93] Moad, G., E. Rizzardo, and S. H. Thang, "Living radical polymerization by the RAFT process - a second update," Aust. J. Chem. 62, 1402-1472 (2009).

[94] Rizzardo, E., M. Chen, B. Chong, G. Moad, M. Skidmore, and S. H. Thang, "RAFT polymerization: Adding to the picture," Macromol. Symp. 248, 104-116 (2007).

[95] Fetters, L. J., D. J. Lohse, and R. H. Colby, Chain Dimensions and Entanglement Spacings (Springer, New York, 2007), pp.447-454.

[96] Van Gurp, M., and J. Palmen, "Time-temperature superposition for polymeric blends," Rheol. Bull. 67, 5-8 (1998).

[97] Trinkle, S., and C. Friedrich, "Van Gurp-Palmen-plot: A way to characterize polydispersity of linear polymers," Rheol. Acta. 40, 322-328 (2001).

[98] Tassieri, M., M. Laurati, D. J. Curtis, D. W. Auhl, S. Coppola, A. Scalfati, K. Hawkins, P. R. Williams, and J. M. Cooper, "i-Rheo: Measuring the materials linear viscoelastic properties 'in a step'!," J. Rheol. 60, 649-660 (2016).

[99] Marín, J. M. R., J. K. Huusom, N. J. Alvarez, Q. Huang, H. K. Rasmussen, A. Bach, A. L. Skov, and O. Hassager, "A control scheme for filament stretching rheometers with application to polymer melts," J. Nonnewton. Fluid Mech. 194, 14-22 (2013).

[100] Zhang, Z., C. Huang, R. Weiss, and Q. Chen, "Association energy in strongly associative polymers,” J. Rheol. 61, 1199-1207 (2017).

[101] Flory, P. J., "Molecular size distribution in three dimensional polymers. I. Gelation," J. Am. Chem. Soc 63, 3083-3090 (1941).

[102] Likhtman, A., "Viscoelasticity and molecular rheology," in Polymer Science: A Comprehensive Reference, 2 nd ed., edited by M. Moeller and K. Matyjaszewski (Elsevier, Amsterdam, 2012), Vol. 1, Chap. 1.06, pp. 133-179.

[103] Ramírez, J., S. K. Sukumaran, B. Vorselaars, and A. E. Likhtman, "Efficient on the fly calculation of time correlation functions in computer simulations," J. Chem. Phys. 133, 154103 (2010).

[104] Doi, M., and S. F. Edwards, The Theory of Polymer Dynamics (Oxford University, New York, 1988).

[105] Dealy, J. M., D. J. Read, R. G. Larson, Structure and Rheology of Molten Polymers: From Structure to Flow Behavior and Back Again (Carl Hanser Verlag GmbH \& Co. KG, München, 2018). 